\title{
LES validation of urban flow, part II: eddy statistics and flow structures
}

Article

Accepted Version

Hertwig, D., Patnaik, G. and Leitl, B. (2017) LES validation of urban flow, part II: eddy statistics and flow structures. Environmental Fluid Mechanics, 17 (3). pp. 551-578. ISSN 1567-7419 doi: https://doi.org/10.1007/s10652-016-9504-x Available at https://centaur.reading.ac.uk/76908/

It is advisable to refer to the publisher's version if you intend to cite from the work. See Guidance on citing.

To link to this article DOI: http://dx.doi.org/10.1007/s10652-016-9504-x

Publisher: Springer

All outputs in CentAUR are protected by Intellectual Property Rights law, including copyright law. Copyright and IPR is retained by the creators or other copyright holders. Terms and conditions for use of this material are defined in the End User Agreement.

\section{www.reading.ac.uk/centaur}

\section{CentAUR}

Central Archive at the University of Reading

Reading's research outputs online 


\title{
LES validation of urban flow, part II: eddy statistics and flow structures
}

\author{
3 Denise Hertwig · Gopal Patnaik · Bernd Leitl
}

5 Received: date / Accepted: date

6 Abstract Time-dependent three-dimensional numerical simulations such as large-eddy sim7 ulation (LES) play an important role in fundamental research and practical applications in meteorology and wind engineering. Whether these simulations provide a sufficiently accurate picture of the time-dependent structure of the flow, however, is often not determined in enough detail.

We propose an application-specific validation procedure for LES that focuses on the time dependent nature of mechanically induced shear-layer turbulence to derive information about strengths and limitations of the model. The validation procedure is tested for LES of turbulent flow in a complex city, for which reference data from wind-tunnel experiments are available. An initial comparison of mean flow statistics and frequency distributions was presented in part I. Part II focuses on comparing eddy statistics and flow structures. Analyses of integral time scales and auto-spectral energy densities show that the tested LES reproduces the temporal characteristics of energy-dominant and flux-carrying eddies accurately.

9 Quadrant analysis of the vertical turbulent momentum flux reveals strong similarities be-

D. Hertwig

Meteorological Institute, University of Hamburg Bundesstrasse 55, D-20146 Hamburg, Germany

Present address:

Department of Meteorology, University of Reading

P.O. Box 243, Reading, RG6 6BB, UK

E-mail: d.hertwig@reading.ac.uk

Tel.: +44-118-378-6721

Fax: +44-118-378-8905

G. Patnaik

Laboratories for Computational Physics and Fluid Dynamics

U.S. Naval Research Laboratory

Washington D.C., USA

E-mail: gopal.patnaik@nrl.navy.mil

B. Leitl

Meteorological Institute, University of Hamburg Bundesstrasse 55, D-20146 Hamburg, Germany

E-mail: bernd.leitl@uni-hamburg.de 
tween instantaneous ejection-sweep patterns in the LES and the laboratory flow, also showing comparable occurrence statistics of rare but strong flux events. A further comparison of wavelet-coefficient frequency distributions and associated high-order statistics reveals a strong agreement of location-dependent intermittency patterns induced by resolved eddies in the energy-production range.

The validation concept enables wide-ranging conclusions to be drawn about the skill of turbulence-resolving simulations than the traditional approach of comparing only mean flow and turbulence statistics. Based on the accuracy levels determined, it can be stated that the tested LES is sufficiently accurate for its purpose of generating realistic urban wind fields that can be used to drive simpler dispersion models.

Keywords Large-eddy simulation · Model validation · Quadrant analysis · Urban environment $\cdot$ Wavelet analysis · Wind tunnel

\section{Introduction}

Time-dependent three-dimensional numerical simulations of turbulent flow originated from meteorological research more than 40 years ago. Having its roots in the development of early numerical weather prediction models $[54,30]$, the first comprehensive applications of largeeddy simulation (LES) in the context of turbulence research were made in the 1970s [14, 50]. With rapidly increasing computational capacities within the last decade or so LES and other computational fluid dynamics (CFD) models became affordable for a broad research community. This development is paralleled by the availability of commercial and opensource codes and toolboxes.

Today, hardly any meteorological or wind engineering research area focusing on mesoscale or micro-scale atmospheric processes is unaffected by the large eddy-resolving approach. LES is frequently applied to study problems in which the time-space evolution of the atmospheric boundary-layer (ABL) is of special interest: e.g. stratification and diurnal transformations of the ABL structure $[35,29,4,12,3]$ or cloud physics $[8,51]$. Another key area of application are flow and dispersion processes in the near-surface atmospheric boundary layer over various surface forms ranging from homogeneous land types over mountainous terrain $[9,33]$ to plant or urban canopies $[52,65,49,6,28]$.

It is increasingly recognised that studying transient (i.e. time-dependent) flow phenomena is at least as important as the time-mean view of turbulence in order to characterise ABL flows. While research on coherent flow structures initially had a strong focus on flow over plant canopies $[42,19]$, scientific interest is continuously shifting towards the connections between organised eddies and turbulent exchange in urban areas. Time-dependent, three-dimensional numerical simulations like LES offer a space/time resolved view on urban turbulence and now play an important role in coherent structure research. The data, if sufficiently quality controlled, can offer an ideal basis for fundamental research. Based on data from direct numerical simulations, Coceal et al. [11], for example, presented a conceptual model describing unsteady urban roughness-sublayer dynamics. Low momentum streaks found above roof level were associated with the passage of hairpin vortices, an eddy class composed of counter-rotating vortex structures that have been extensively studied in flat-wall boundary-layer flows [43,2]. A second flow regime evolves in the shear layer on top of the canopy. In this region, large-scale eddies are generated by the rolling-up of shear zones and intermittent vortex shedding from rooftops. These structures travel downstream, impinge on other buildings, and may interact with recirculation patterns in street canyons. 
Within the urban canopy layer (UCL) Coceal et al. [11] found inclined vortex structures with characteristic vorticity patterns that are of great importance for urban flow dynamics, particularly regarding their influence on momentum, heat and pollutant transport.

\subsection{Validation concept}

The time-dependent nature of LES complicates the assessment of the quality of the prediction and makes thorough validation of time-resolved simulations challenging. If conducted at all, comparisons between LES and reference data, e.g. from experiments, are usually restricted to mean flow and turbulence statistics. Strictly speaking, this only provides sufficient insight about the accuracy of models based on the averaged conservation equations like the Reynolds-averaged Navier-Stokes (RANS) equation models. In the case of turbulenceresolving simulations such as LES, however, a thorough validation should also assess the degree to which the code captures the transient structure of the flow. Established methods from the field of signal analysis and flow pattern recognition can open up new ways to define quality criteria by which to assess the model output.

In part I we introduced a holistic LES validation concept for near-surface atmospheric flow based on a sequence of well-established time-series analysis methods. The essential premise here is that the time-dependent nature of LES has to be taken into account for the validation to provide a true assessment of the capabilities and limitations of the model. The proposed validation hierarchy distinguishes three comparison levels:

1. Exploratory data analysis (here: descriptive statistics, frequency distributions)

2. Analysis of turbulence scales (here: temporal autocorrelations, energy density spectra)

3. Flow structure identification (here: quadrant analysis, continuous wavelet transform).

The three levels offer increasingly deeper insight into the simulation properties in terms of the representation of turbulence structures, but also make increasingly higher demands on the quality and quantity of reference data.

\subsection{Test scenario, data and study layout}

We test the validation approach based on a particularly challenging scenario: turbulent flow in a densely built-up urban centre. A detailed account of relevant information about the test scenario, the LES and the laboratory experiment was presented in part I. For the sake of brevity, we only provide an overview here.

The high-density city centre of Hamburg, Germany, serves as the test bed for the validation exercise. The inner city is characterised by an average building height of $H=34.3 \mathrm{~m}$ with typical street canyon widths in the order of $W=20 \mathrm{~m}$. This results in a typical streetcanyon aspect ratio in the inner city of $H / W=1.72$. The LES tested is the urban aerodynamics code FAST3D-CT [40,39], developed and operated by the U.S. Naval Research Laboratory. The code uses an implicit representation of subgrid-scale turbulence, which is a very efficient approach for LES in large urban domains with high spatial resolution. In this study the simulation was run with a spatial resolution of $2.5 \mathrm{~m}$ within the urban roughness sublayer in a $4 \mathrm{~km} \times 4 \mathrm{~km}$ computational domain. The purpose of this particular simulation was the provision of realistic urban wind data that can be used off-line to drive an urban plume dispersion model. 


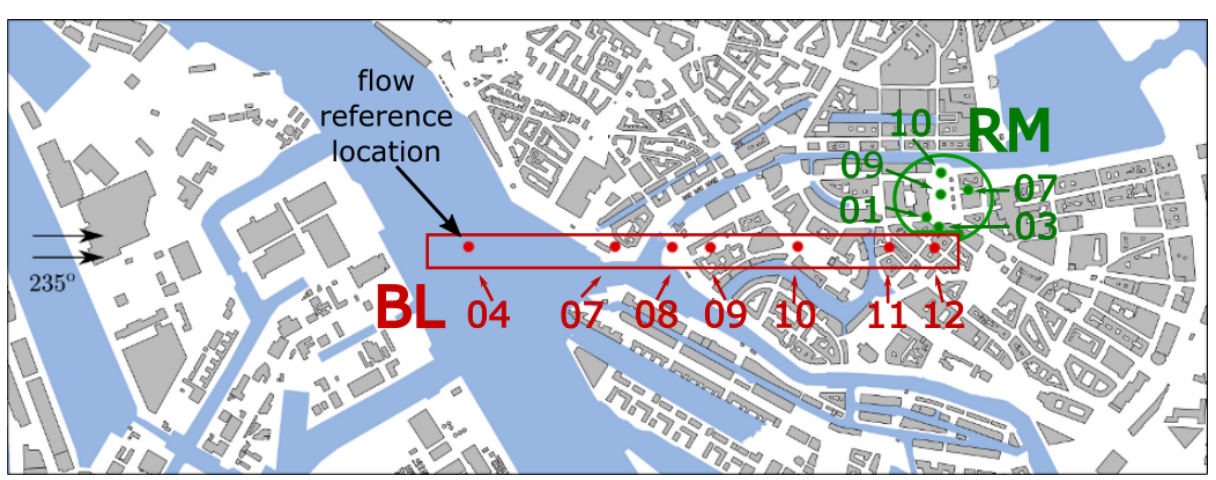

Fig. 1: Wind-tunnel model area indicating the boundary-layer development positions (prefix $B L$, red dots) and selected sites around the city hall (prefix $R M$, green dots). The Elbe river separates the high-density city centre to the north from the low-rise industrial harbour region. The flow reference location above the river (BL04) is also indicated.

Point-wise velocity time series were extracted at cell centres every $0.5 \mathrm{~s}$ over a duration of 23,250 s (approx. $6.5 \mathrm{~h}$ ). Time-resolved, point-wise reference data have been generated using laser Doppler anemometry (LDA) in a boundary-layer wind tunnel based on a detailed scale representation of the urban test area (geometric scale: 1:350). The longitudinal/lateral extents of the wind-tunnel model were $3.7 \mathrm{~km} / 1.4 \mathrm{~km}$ full-scale $(10.5 \mathrm{~m} / 4 \mathrm{~m}$ in model scale). The model area is shown in Fig. 1 together with the locations of the comparison sites that is focused on in the following sections (for a detailed discussion of local geometry and flow pattern at these sites see part I). With the LDA system, two velocity components ( $U-V$ or $U-W$ pairs) were simultaneously measured over a duration of $170 \mathrm{~s}(16.5 \mathrm{~h}$ under full-scale conditions). When operated in $U-W$ mode, the LDA measuring volume and the probe itself are aligned at the same height. In order to avoid physical interferences with model buildings, measurements of the vertical velocity component were only conducted at locations above the UCL $(z \geq 1.2 H)$ at the BL sitess. For time series analyses the discontinuous LDA signals were reconstructed using a sample-and-hold technique.

In the first part of the study we covered the initial level of the validation concept by comparing mean flow and turbulence statistics. This was extended by the analysis of the underlying velocity time series in terms of frequency distributions, which documented the strength of the tested code to capture characteristic urban flow features.

In the following, we focus on the comparative analysis of turbulence scales and transient flow patterns in the urban roughness sublayer. The analysis methods used in this study are introduced in Sect. 2. We then follow the second and third levels of the validation strategy by comparing temporal auto-correlations, turbulence integral time scales and spectral energy densities (Sect. 3) and apply flow pattern recognition techniques (conditional resampling and joint time frequency analyses) in Sect. 4. The fitness of the proposed methodology for a detailed LES validation is discussed in Sect. 5 along with final conclusions and recommendations for next steps. 


\section{Analysis methods}

In the following we present an overview of the methods applied to analyse and compare eddy statistics and flow patterns based on single-point time-resolved velocity signals. A fixed Cartesian model coordinate system $(x, y, z)$ is used and the corresponding streamwise (longitudinal), spanwise (lateral) and vertical components $U_{i}(i=1,2,3)$ of the velocity vector are denoted as $U, V$ and $W$. Overbars denote time-averaged quantities. Velocity statistics are non-dimensionalised based on the mean streamwise reference velocity $U_{\text {ref }}$ at a reference height of $z_{\text {ref }}=45.5 \mathrm{~m}(1.33 \mathrm{H})$ above ground upstream of the inner city area (Fig. $1)$.

\subsection{Integral time scales and energy spectra}

Turbulence integral time scales can be determined from velocity fluctuation time series based on temporal autocorrelations. For stationary flow the 1D autocorrelation function of the fluctuating $i$ th velocity component, $u_{i}^{\prime}=U_{i}-\bar{U}_{i}$, with $u_{1}^{\prime}=u^{\prime}, u_{2}^{\prime}=v^{\prime}, u_{3}^{\prime}=w^{\prime}$, as a function of time lag $t_{l}$ is given by

$$
R_{i i}\left(t_{l}\right)=\frac{1}{\sigma_{i}^{2}} \overline{\left(U_{i}(t)-\bar{U}_{i}(t)\right)\left(U_{i}\left(t+t_{l}\right)-\bar{U}_{i}\left(t+t_{l}\right)\right)} .
$$

$R_{i i}$ describes the degree of common variation in a variable depending on the time difference between two observations, and hence is a measure of the flow memory [55,31]. Motions separated by sufficiently long lags become statistically independent and $R_{i i} \rightarrow 0$ [57]. The integral time scale $\tau_{i i}$ is obtained from

$$
\tau_{i i}=\int_{t_{l_{0}}}^{t_{l_{\infty}}} R_{i i}\left(t_{l}\right) d t_{l}
$$

Different specifications of the upper integration limit $t_{l_{\infty}}$ can be used, e.g. as the zerocrossing point of $R_{i i}$ or as the time after which $R_{i i}$ has dropped below a critical value [38]. The computations can become ambiguous in cases where $R_{i i}$ does not decrease monotonically, but instead oscillates or does not drop to zero within the maximum recorded time interval. The physical relevance of such oscillations is debatable [64]. In this study it is assumed that they reflect the increasingly uncertain nature of $R_{i i}$ at large time lags (i.e. smaller sample sizes). This is supported by the fact that the intensity of the tail oscillations is related to the overall duration of the signal and hence to the statistical representativeness obtained from the respective averaging times. In order to consistently derive $\tau_{i i}$ we use an extrapolation approach for the tails [20]. While the bulk of the original $R_{i i}$ function is preserved at small time lags, the curvature of its tail is approximated by an exponential decay (Fig. 2). The upper integration limit $t_{l_{\infty}}$ is then defined as the time after which $R_{i i}^{f i t} \leq 0.01$.

Spectral energy-density functions $E_{i i}(f)$ provide information about the distribution of the signal's variance among different eddy scales. Due to the component resolution of the experimental data available in this study, the analysis concentrates on the comparison of 1D spectra and co-spectra of two velocity components. Fourier coefficients $\widehat{u}_{i}(f)$ of velocity fluctuations $u_{i}^{\prime}(t)$ are derived from a fast Fourier transform (FFT) based on the CooleyTukey algorithm [13]. Taking the example of the streamwise fluctuations, the one-sided auto-spectral energy densities are obtained according to 

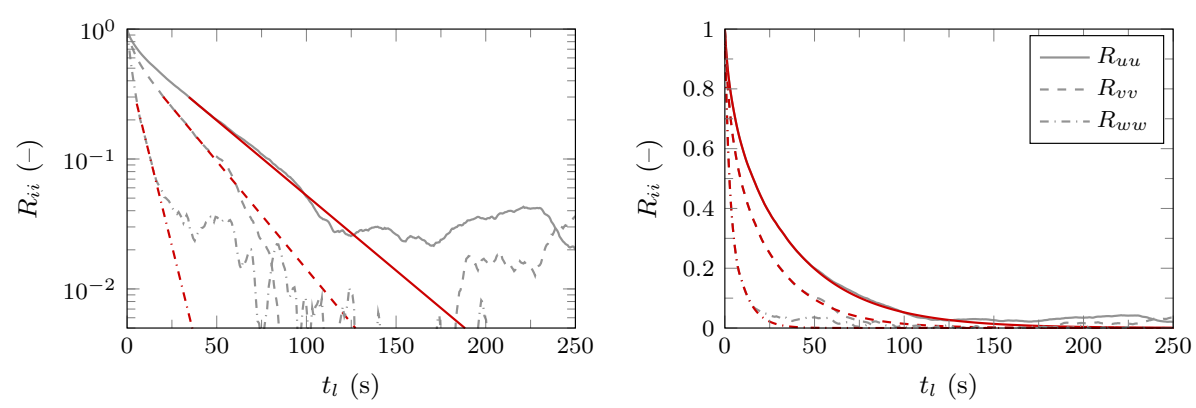

Fig. 2: Example of the fitting procedure applied to the $R_{i i}$ functions (here: wind tunnel data). Left: original curves together with the respective tail fit; right: concatenated signals using the original and fitted curves. The original curves are retained up to time lags for which $R_{i i}$ has dropped to a value between once or twice its $e$-folding time.

$$
E_{u u}\left(f_{k}\right)=\frac{2}{N f_{s}} \widehat{u}_{k}^{*} \widehat{u}_{k}=\frac{2}{N^{2} \delta f_{s}}\left|\widehat{u}_{k}\right|^{2}
$$

with the frequency index $k=0, \ldots, N / 2, N$ is the number of samples in the signal, $f_{s}$ is the sampling frequency, $\delta f_{s}=f_{k}-f_{k-1}$ is the constant frequency increment and the asterisk denotes the complex conjugate [36]. In the same way energy density spectra $E_{v v}$ and $E_{w w}$ can be derived from spanwise and vertical velocity fluctuations, $v^{\prime}$ and $w^{\prime}$. For paired signals of $u^{\prime}$ and $w^{\prime}$ the co-spectrum $\operatorname{Co}_{u w}$ is computed according to $\operatorname{Co}_{u w}\left(f_{k}\right)=$ $\operatorname{Re}\left\{\widehat{u}_{k}\right\} \operatorname{Re}\left\{\widehat{w}_{k}\right\}+\operatorname{Im}\left\{\widehat{u}_{k}\right\} \operatorname{Im}\left\{\widehat{w}_{k}\right\}$.

Earlier studies have shown that the unique roughness structure of urban surfaces leaves a distinct footprint in the spectra $[45,46]$. Inertial subrange behaviour in urban areas in terms of $-5 / 3$ slopes is comparable to flow over uniform roughness. However, based on a more stringent test for local isotropy, Rotach [45] found that urban flow is not truly isotropic in the inertial subrange at heights well within the roughness sublayer. The size of integral length scale eddies associated with the spectral peaks deviates from empirical reference relations for flow over homogeneous surfaces [25]. Roth [46] reported that within the UCL and in the vicinity of the canopy top, a shift towards higher frequencies is evident in the spectra of the horizontal velocities, while peaks in vertical velocity spectra are offset towards lower frequencies. The increase of the vertical eddy-length scale suggests that the vertical transport is dominated by wake turbulence that scales with the building dimensions.

\subsection{Conditional averaging}

A well-known representative of conditional resampling/averaging methods is the quadrant analysis $[60,61,59]$, which can be applied, for example, to analyse the vertical turbulent momentum exchange $\overline{u^{\prime} w^{\prime}}$. Instantaneous fluxes $u^{\prime} w^{\prime}$ are grouped into one of four quadrants based on the respective composition of the algebraic signs of $u^{\prime}$ and $w^{\prime}$. Following the notation by Raupach [41], conditional averages of the momentum flux contributions from each of the four quadrants are obtained from 


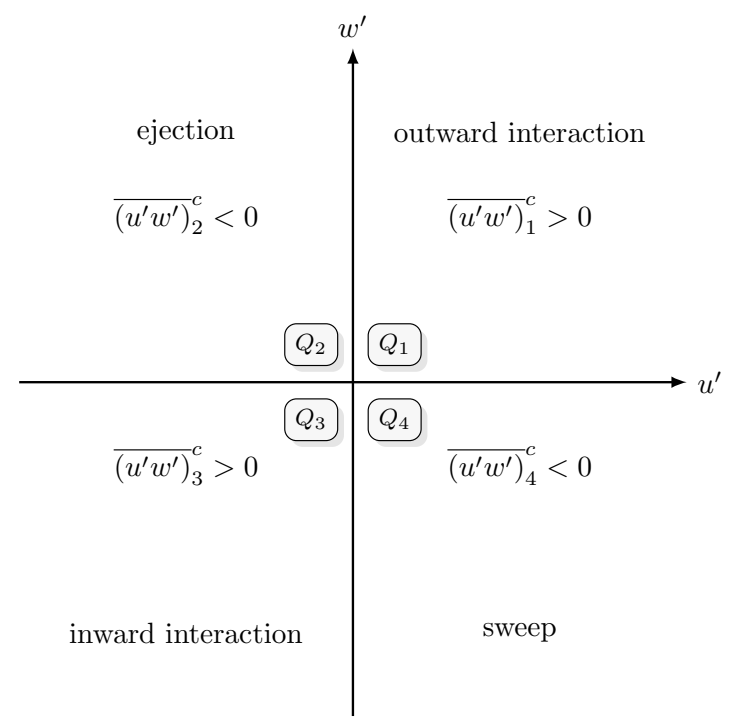

Fig. 3: The four quadrants of the vertical turbulent momentum flux $u^{\prime} w^{\prime}$.

$$
{\overline{\left(u^{\prime} w^{\prime}\right.}}_{i}^{c}=\lim _{T \rightarrow \infty} \frac{1}{T} \int_{0}^{T} u^{\prime}(t) w^{\prime}(t) I_{i}(t) d t
$$

where $c$ is a reminder for the conditional nature of the time-average, $T$ is the signal duration, and $I_{i}(t)$ is a trigger function yielding 1 if $u^{\prime}(t)$ and $w^{\prime}(t)$ are in the ith quadrant and 0 otherwise. The relative contributions from individual quadrants to the total flux $\overline{u^{\prime} w^{\prime}}$ are measured in terms of flux fractions $S_{i}={\overline{\left(u^{\prime} w^{\prime}\right.}}_{i}^{c} / \overline{u^{\prime} w^{\prime}}$, where $\sum_{i} S_{i}=1$.

Fig.3 shows a schematic of the quadrant separation of the instantaneous momentum flux $u^{\prime} w^{\prime}$. In the first and third quadrant, $Q_{1}$ and $Q_{3}, u^{\prime}$ and $w^{\prime}$ are of the same sign and conditional averaging results in positive fluxes ${\overline{\left(u^{\prime} w^{\prime}\right)}}_{1,3}^{c}>0$. Corresponding fluid motions are denoted as outward and inward interactions [60]. The second quadrant, $Q_{2}$, is associated with the upward ejection of momentum $\left(u^{\prime}<0, w^{\prime}>0\right)$. The opposing process is the downward sweep of momentum $\left(u^{\prime}>0, w^{\prime}<0 ; Q_{4}\right)$. Conditionally averaging over ejection and sweep events results in negative fluxes $\frac{\left(u^{\prime} w^{\prime}\right)_{2,4}^{c}}{c}<0$. The difference $\delta S_{4,2}=S_{4}-S_{2}$ quantifies the local dominance of ejection or sweep contributions. The flux exuberance $E x=\left(S_{1}+\right.$ $\left.S_{3}\right) /\left(S_{2}+S_{4}\right)$ is a measure of the relative importance of organised gradient motions $\left(Q_{2,4}\right)$ over counter-flux events $\left(Q_{1,3}\right)$ for the local flux balance $[53,10]$.

The instantaneous fluxes $u^{\prime} w^{\prime}$ can by far exceed the magnitude of $\overline{u^{\prime} w^{\prime}}$. To determine the relative importance of large amplitude contributions to the flux fractions, Willmarth and Lu [61] proposed to analyse fluctuation combinations that exceeds a specified threshold. This approach is known as hole-size analysis and allows the comparison of occurrence probabilities of extreme flux events in LES and experiment. Following Raupach [41], the successive filtering of extreme events is implemented by adding another constraint on the trigger function $I_{i}$ by only averaging over fluxes for which $\left|\left(u^{\prime} w^{\prime}\right)_{i}\right| \geq H_{c}\left|\overline{u^{\prime} w^{\prime}}\right|$, with $H_{c}$ being the hole size $\left(H_{c}=0,1,2, \ldots, 30\right.$ in this study). 
Within and above the UCL building-induced turbulence can induce strong turbulent mixing [46]. Through the analysis of the vertical momentum flux, the relative contributions of the upward transport of momentum deficit (ejection) and the downward transport of momentum excess (sweep) in urban environments can be investigated [44]. At roof-level, the momentum exchange is often found to be dominated by sweeps. However, this prevalence vanishes at higher elevations. The dominance of sweeps within the UCL has been confirmed on the basis of field observations [37,10], showing that ejections are prevailing well above the canopy. Oikawa and Meng [37] described characteristic sweep and ejection patterns associated with sudden fluid bursts and connected distinctive ramp structures in temperature signals with the passage of large-scale coherent eddies above the canopy. Based on conditional averages of ejection-sweep cycles within and above a street canyon, Feigenwinter and Vogt [18] showed that fluctuation levels were highest just above the canopy and decreased with increasing distance from the roofs. Christen et al. [10] described the role of coherent structures for turbulent exchange at the interface between canopy and roughness sublayer by associating ejection-sweep events with the advection and penetration of coherent structures from the roughness layer into the street canyon.

\subsection{Joint time-frequency analysis}

The wavelet transform is an important representative of joint time-frequency analysis methods used for the time-localisation of a signal's frequency content [34,22,23]. In effect, this approach adds the time dimension to the classic Fourier analysis by using wave functions of limited temporal support instead of non-local sinusoids. In the application to turbulent flows this means that the occurrence of eddy structures associated with certain frequencies can be studied in a time-dependent framework [32,15].

Wavelets are oscillating, square-integrable, localised functions whose location and shape are manipulated during the transform process to unfold the time-frequency content of the signal [1]. The wavelet function $\psi_{s, n}(t)=s^{-1 / 2} \psi\left[(t-n) s^{-1}\right]$ depends on two parameters. The translation parameter $n$ shifts the wavelet along the time axis and the dilation parameter $s>0$, also known as the scale of the wavelet, stretches $(s>1)$ or compresses $(0<s<1)$ the function in order to retrieve low or high frequency information from the signal. This behaviour is illustrated in Fig. 4. The normalisation factor $s^{-1 / 2}$ ensures finite energy content at all wavelet scales [26]. The continuous wavelet transform (CWT) of a time-dependent signal $\Phi(t)$ with zero mean and finite energy is given by

$$
W_{n}(s)=\frac{1}{\sqrt{s}} \int_{-\infty}^{\infty} \Phi(t) \psi^{*}\left(\frac{t-n}{s}\right) d t,
$$

where the asterisk denotes the complex conjugate. The wavelet coefficients $W_{n}(s)$ contain time-frequency information about $\Phi(t)$. The time-frequency resolution of the CWT is variable. At large scales, the wavelet is less well localised in time than at small scales, while the frequency resolution is better than for contracted wavelets (Fig. 4). Like the Fourier transform, the CWT is reversible and the original signal can be reconstructed from the wavelet coefficients without information loss [36].

For our analyses of velocity time series we use the complex Morlet wavelet defined as $\psi_{m}(t)=N_{\psi_{m}} \exp \left(i \omega_{0} t\right) \exp \left(-t^{2} / 2\right)$. The central frequency $\omega_{0}$ is set to a value of 6 in this study, and $N_{\psi_{m}}=\pi^{-1 / 4}$ is a normalisation factor. We apply a discretised version of the CWT in spectral space [1,56]. For a discrete signal $\Phi_{n}=\Phi\left(t_{n}=n \delta t_{s}\right)$ sampled at time intervals 


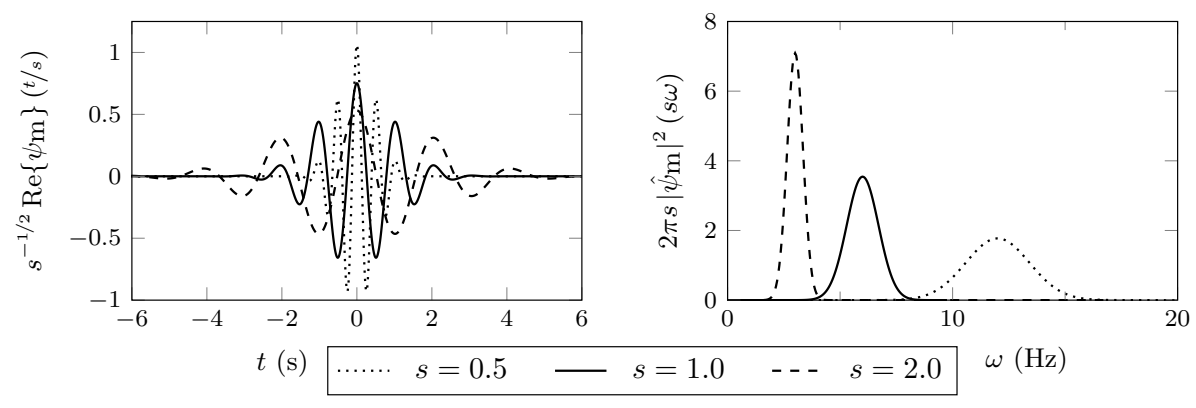

Fig. 4: Influence of the dilation parameter $s$ on the shape of the Morlet wavelet in the time (left) and frequency domain (right), with the latter showing the wavelet's energy spectrum.
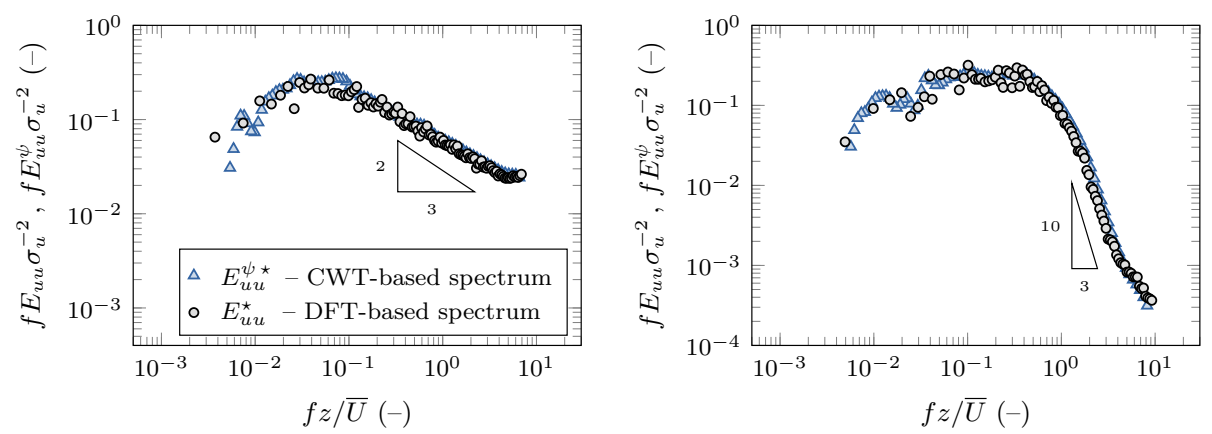

Fig. 5: Scaled auto-spectral energy densities of the streamwise velocity derived from the discrete-time CWT using the Morlet wavelet (triangles) in comparison to the classic discrete Fourier transform (DFT) spectra (dots). Left: wind tunnel; right: LES. The spectra correspond to a height of $45.5 \mathrm{~m}$ above the Elbe river (site BL04).

$\delta t_{s}$ over a duration of $T=N \delta t_{s}$, where $N$ is the number of samples and $n=0, \ldots, N-1$, the discrete-time CWT is given by

$$
W_{n}(s)=\sqrt{\frac{2 \pi s}{\delta t_{s}}} \sum_{k=0}^{N-1} \widehat{\Phi}_{k} \hat{\psi}^{*}\left(s \omega_{k}\right) \exp \left(i \omega_{k} n \delta t_{s}\right),
$$

where $\widehat{\Phi}$ and $\hat{\psi}$ are the Fourier transforms of the signal and the wavelet, respectively, $k$ is a frequency index and $\omega=2 \pi f$. The term before the sum is a normalisation factor that ensures that the wavelet function has unit energy at each scale. The Fourier transform of the Morlet wavelet is known analytically: $\hat{\psi}_{\mathrm{m}}(\omega)=N_{\psi_{\mathrm{m}}} \mathscr{H}(\omega) \exp \left(-\left(\omega-\omega_{0}\right)^{2} / 2\right)$, where $\mathscr{H}$ is the Heaviside step function [56]. Eq. (6) is implemented by obtaining the inverse Fourier transform of the product of $\widehat{\Phi}_{k}$ and $\hat{\psi}^{*}\left(s \omega_{k}\right)$ for all scales $s$ at all translations $n$ using an FFT algorithm [13]. Following [56], the series of scales $s_{j}$ is obtained as a fractional power of 2 according to $s_{j}=s_{0} 2^{j \delta j}$, where $s_{0}$ is the smallest scale and $\delta j$ is the spacing between scales. In this study we use $s_{0}=\delta t_{s}$ and $\delta j=1 / 8$ following sensitivity tests.

As the energy content of the signal is conserved in wavelet space, it is possible to obtain a global energy density spectrum based on $W_{n}(s)$ similar to the spectrum available from the 
discrete Fourier transform [1]. Fig. 5 shows wind tunnel and LES energy-density spectra corresponding to a height of $45.5 \mathrm{~m}$ above the Elbe river derived from a discrete-time CWT using the complex Morlet wavelet in comparison to the classic Fourier spectra. Both, the $-2 / 3$ slope of the wind-tunnel inertial subrange as well as the much steeper slope of the LES spectrum (approximately $-10 / 3$ as a result of cutting off eddies smaller than the numerical grid) are very well resolved with the CWT. This fast energy decay is characteristic for LES spectra due to the spatial filtering and can only be adequately resolved with wavelets that have a high number of vanishing moments. With the Morlet wavelet using $\omega_{0}=6$ spectral slopes up to -7 can be reproduced [16].

\section{Eddy statistics}

In the following sections we present results of the validation test case based on detailed analyses of velocity time series. Results are only directly compared at heights for which the largest spatial offset between the LES and wind-tunnel data pairs was $0.25 \mathrm{~m}$. This offset is well within the LDA's spatial accuracy of $0.56 \mathrm{~m}$ full scale in $z$-direction when operated in $U-V$ mode or in $y$-direction for $U-W$ measurements (see part I for details).

The validation results presented in the following paragraphs and in Sect. 4 represent only a subset of the analyses performed in the course of the validation study in order to focus on particular strengths and limitations of the model. The selection is representative of the overall agreement between experiment and LES.

\subsection{Integral time scales}

Integral time scales can be regarded as representative time scales of the dominant turbulence structures in the flow. Comparing their characteristics is therefore particularly important for eddy-resolving approaches such as LES.

In the following, comparisons of $\tau_{i i}$ and $R_{i i}$ are presented in full-scale dimensions. The full-scale time lags $t_{l}$ used to construct $R_{i i}\left(t_{l}\right)$ were derived from their dimensionless equivalents $t_{l}^{\star}$ according to $t_{l}^{\star}=t_{l} U_{r e f} L_{r e f}^{-1}$, setting $U_{\text {ref }}$ to $5 \mathrm{~m} \mathrm{~s}^{-1}$ and using reference lengths, $L_{r e f}$, of $1 \mathrm{~m}$ for the laboratory flow and an equivalent of $350 \mathrm{~m}$ for the LES.

\subsubsection{Vertical structure of $\tau_{i i}$}

Fig. 6 shows height profiles of Eulerian integral time scales for the three velocity components within the roughness sublayer up to approximately $2 \mathrm{H}$ at four of the BL comparison sites, for which measurements of all three velocity component are available from the reference experiment. The statistical reproducibility of the experimental results was derived from repetition measurements, yielding full-scale maximum scatter of $\tau_{11} \pm 3.95 \mathrm{~s}, \tau_{22} \pm 1.85 \mathrm{~s}$, and $\tau_{33} \pm 1.17 \mathrm{~s}$.

Overall, the LES captures the qualitative height-dependence of the integral time scales at most of the sites. While there are some quantitative differences, the overall magnitude of turbulence time scales agrees with the experiment. A steady increase of $\tau_{i i}$ is seen well above roof level, corresponding to an increase of eddy length scales in response to the gradual weakening of topology-induced flow effects. However, at site BL07 the decrease of the LES $\tau_{11}$ and low values of $\tau_{33}$ above $H$ is opposite to the behaviour seen in the experiments. This site is located in the region where an internal boundary layer is starting to develop just 

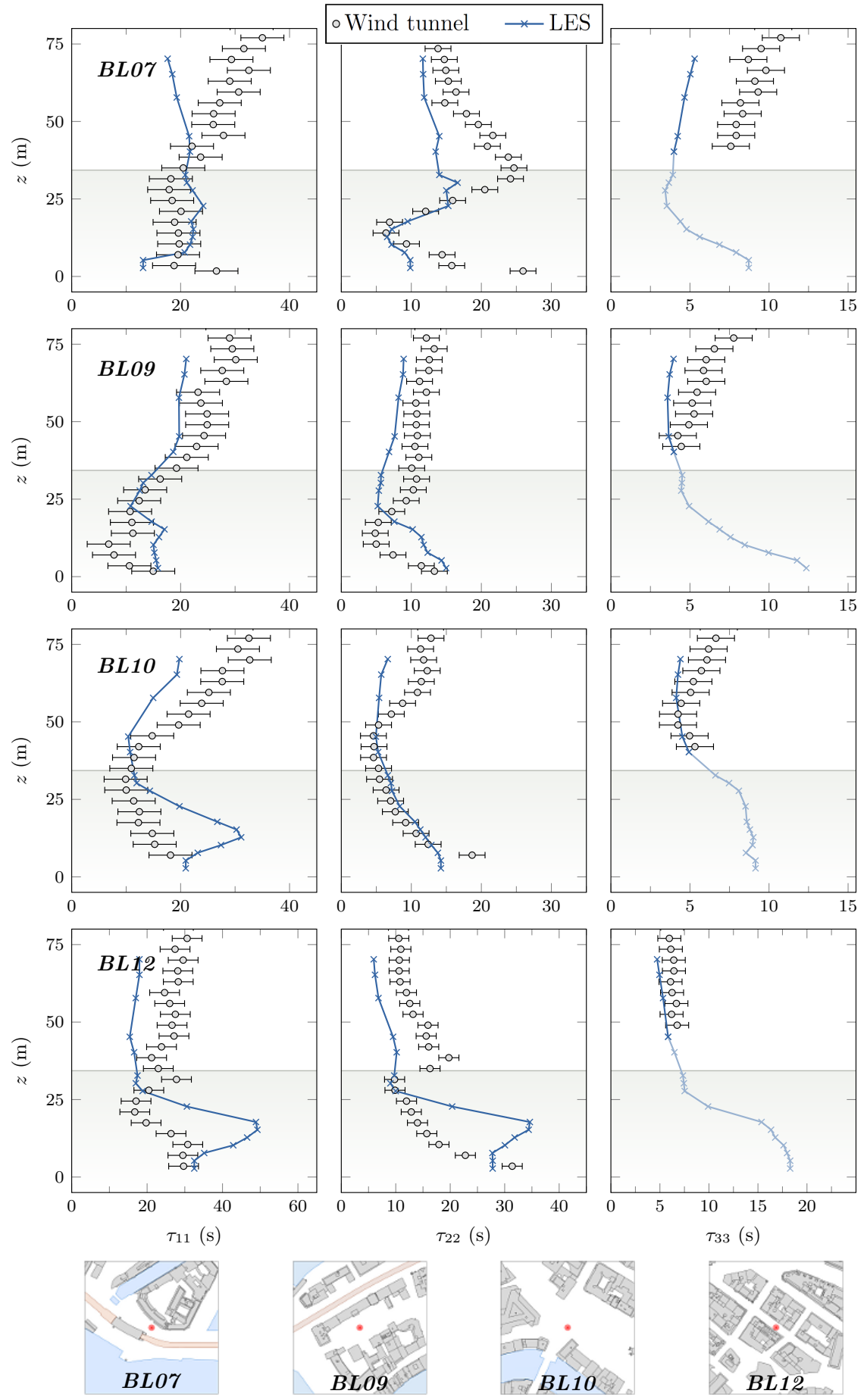

Fig. 6: Comparison of height profiles of $\tau_{11}$ (left column), $\tau_{22}$ (centre) and $\tau_{33}$ (right) at four comparison locations (wind tunnel: dots; LES: crosses). The grey shading indicates heights lower than the mean building height of $H=34.3 \mathrm{~m}$ in the city centre. Maps showing the location of the comparison points depict an area of $210 \mathrm{~m} \times 210 \mathrm{~m}$. Times are given in full scale. 
downstream of the river in response to the increased surface roughness of the high-density inner city. Here it seems that the upper layer flow field in the LES still is dominated by the artificial turbulence prescribed at the inflow plane of the simulation. Further downstream, as the flow adjusts to the new roughness underneath, the simulation above the UCL becomes more self-consistent, resulting in an increased level of agreement with the wind tunnel.

The same is true for the height development of integral time scales $\tau_{33}$ associated with the vertical velocity from site BL07 to BL12. At elevations at which measurements are available, the $\tau_{33}$ of the LES are well within the experimental scatter at most heights in the downtown area (BL09-BL12). Within the UCL the LES predicts increased amplitudes of $\tau_{33}$ (BL09, BL12), indicating enhanced memory effects associated with the vertical momentum exchange, for example associated with street-canyon ventilation at site BL12. Although direct validation of this simulation feature is not possible here due to the lack of experimental data points, the agreement of the magnitudes of $\tau_{11}$ and $\tau_{22}$ indicates that this is also true for $\tau_{33}$ for consistency reasons.

The overall height structure of $\tau_{i i}$ uniquely corresponds to the local building topology and thus changes strongly from site to site. For example, at sites BL10 (intersection) and BL12 (street canyon) strong vertical gradients in $\tau_{11}$ and $\tau_{22}$ can be observed within the UCL; a feature that is more pronounced in the LES. At all sites the wind-tunnel flow is characterised by long $\tau_{22}$ well within the UCL, indicating the existence of comparatively long-lived eddy structures. These features are also evident in the LES, although the peak heights and magnitudes differ at some of the locations, e.g. BL12.

By comparing integral time scales at various sites, it could be shown that the LES responds in a similar way to the local building morphology as the flow in the reference experiment. Here it is particularly important to analyse all three components of the velocity vector, as the urban flow field is highly three-dimensional and the LES should be able to reflect this complexity. This is crucial, for example, for the representation of horizontal and vertical mixing in turbulent dispersion processes. The results demonstrate the ability of the tested LES code to represent the time-scales of energy-dominating turbulence features realistically for the given application, even in a very complex geometry and with limitations imposed by the grid resolution.

\subsubsection{Structural information from $R_{i i}$}

It is worthwhile to also investigate the shapes of the underlying temporal autocorrelation curves, $R_{i i}\left(t_{l}\right)$ in order to derive further information about eddy structures.

Two commonly observed features are shown in Fig. 7, depicting close-ups of LES and wind tunnel data at short time lags. Strikingly different curvatures of the LES and laboratory autocorrelations can be observed at $t_{l} \leq 10 \mathrm{~s}$. While the experimental curves are moreor-less straight lines, indicating a fast exponential decay (note the use of logarithmic $y$ axes), the eddies in the LES are slightly longer correlated over short times. This feature is characteristic of the spatially filtered nature of the LES, in which only the large eddies are directly resolved. As discussed by Townsend [57], in turbulent flows in which eddy sizes are in some way restricted the slope of the $R_{i i}$ functions is rather gentle at short time lags. If, on the other hand, a wide and continuous range of eddy structures is present in the flow, as is the case in the wind tunnel, the initial slope is significantly steeper.

Another noticeable feature encountered at various locations in the urban domain is that some of the autocorrelation functions are composed of rapidly and slowly varying parts, a feature that is often more pronounced in the autocorrelations of the streamwise velocity fluctuations. The example of location RM10 (Fig. 7, left) shows that in both data sets the 


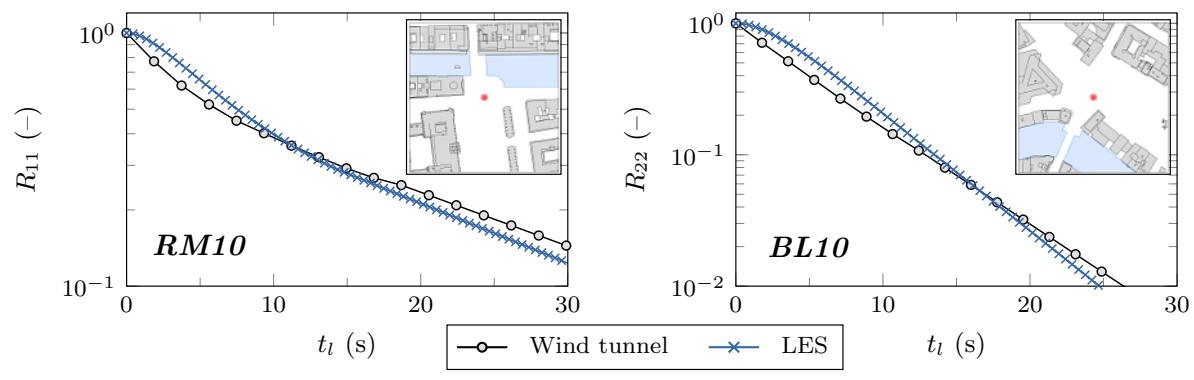

Fig. 7: Autocorrelation functions at two example locations in the inner city for the wind tunnel (dots) and the LES (crosses). Left: $R_{11}$ at location RM10 in a height of $40.25 \mathrm{~m}$ $(1.2 H)$; right: $R_{22}$ at location BL10 in a height of $28 \mathrm{~m}(0.8 \mathrm{H})$. Time lags are presented in full scale.

$R_{11}$ slopes are clearly steeper for time lags below $10 \mathrm{~s}$, approximately corresponding to the $e$-folding time of the functions, than for the remaining time periods. This is an indication of the superposition of two autocorrelation functions and associated dominant eddy time scales (e.g. discussions in [57,31]). Due to the complexity of urban flow fields, at certain positions the flow may locally be dominated by more than one regime of turbulent motions with different length and time scales of associated eddy classes.

\subsection{Energy density spectra}

Energy density spectra are utilised to comparatively evaluate the distribution of energy among eddies present in the flow and to compare frequency bandwidths corresponding to spectral energy peaks, which have a direct relation to $\tau_{i i}$. From LES it is expected that energy-containing turbulence is directly resolved, ideally well into the inertial subrange. Formally, the effective resolution of the LES is coupled to the grid size, which acts as a spatial filter, and to the properties of the employed numerical methods. In addition, the nature of the flow has an influence on local resolution characteristics. The overall length scales of the energy-dominating eddies within the UCL, for example, are smaller than those encountered in the inertial sublayer. In the LES code evaluated here only structures sufficiently larger than $2.5 \mathrm{~m}$ are directly resolved. Hence it needs to be evaluated whether this grid resolution is sufficient to resolve even the comparatively small-scale energy-dominating eddy ranges associated with typical canopy-layer turbulence.

Fig. 8 (first three rows) shows examples of the local agreement between wind tunnel and LES auto-spectra above the mean building height $(1.3 H)$. As is apparent from these plots, in the densely built-up city centre the mean LDA sampling rates achieved in the wind tunnel were too low to fully resolve the inertial subrange portion of the flow. Due to enhanced mixing in the urban roughness sublayer the LDA particle seeding is more homogeneous, but less dense on average than in the approach flow. Nevertheless, at all locations the time-resolution of the experimental data is sufficient to directly compare with the energy-containing spectral ranges resolved by the LES.

For all velocity components and all comparison points the agreement between the LES and wind-tunnel spectra is remarkably good in the low-frequency range that is associated with large, anisotropic eddies. The spectral peak regions agree well over the range of mor- 

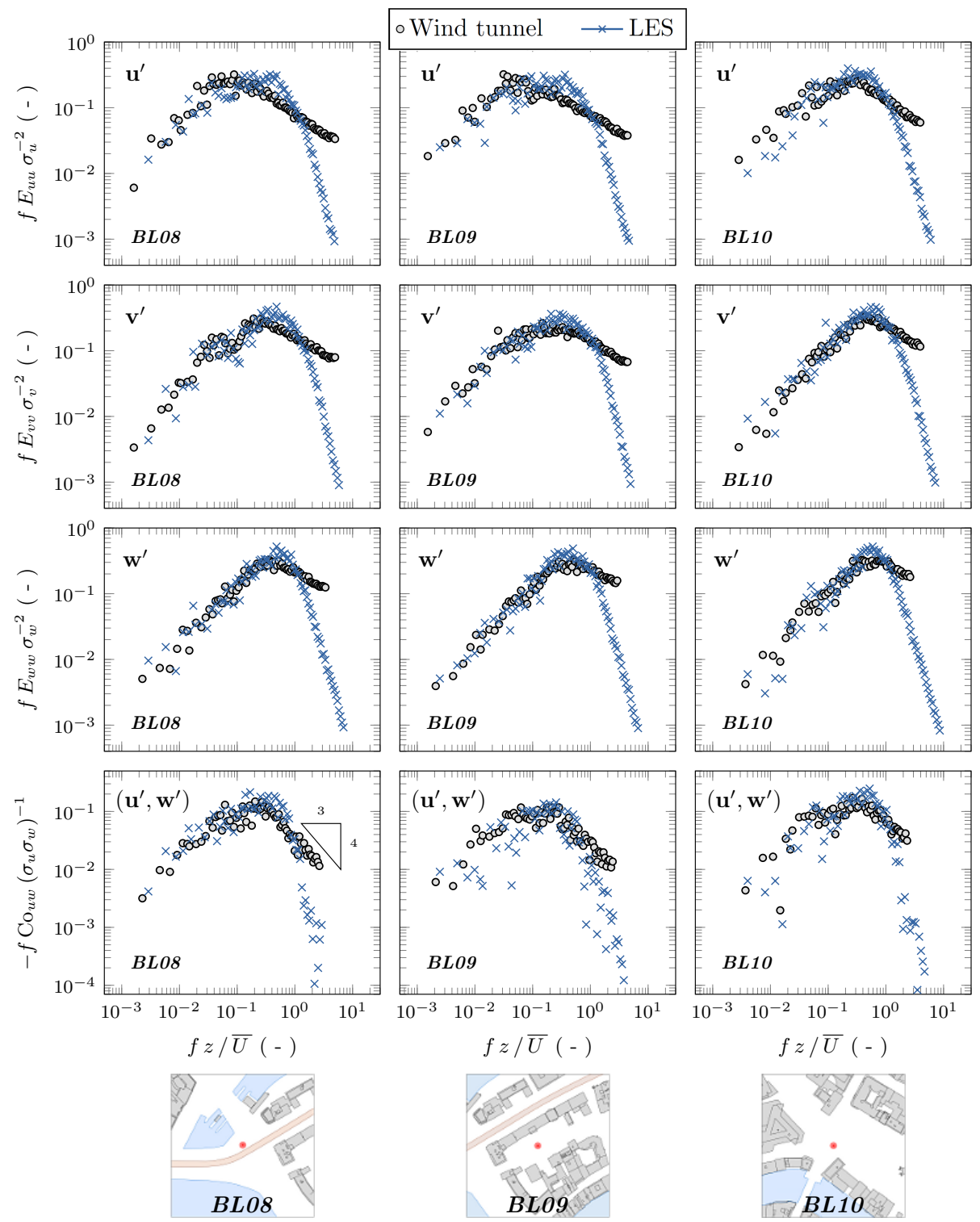

Fig. 8: Comparison of wind tunnel (dots) and LES (crosses) auto-spectral energy densities and co-spectra (bottom row) at three different locations in a height of $1.3 \mathrm{H}(45.5 \mathrm{~m})$. The respective velocity component analysed, i.e. $u^{\prime}, v^{\prime}, w^{\prime}$ or $\left(u^{\prime}, w^{\prime}\right)$, is indicated in the plots. The spectra are presented in a referenced framework based on the mean streamwise velocity $\bar{U}$ and the velocity variance $\sigma_{i}^{2}$ at height $z$. Maps show the location of the comparison sites and their immediate surroundings. 
phologically rather different comparison sites. The fast roll-off of the numerical spectra at high frequencies is quite apparent, starting approximately after one decade into the inertial subrange. An interesting double-peak pattern in the $v^{\prime}$-spectra is evident at site BL08 in both the laboratory and the simulation, implying a structural change in the flow. While the first peak corresponds to the peak frequency range characteristic for the approach flow (see BL04 spectra shown in Fig. 5), the second peak agrees well with the frequencies determined further downstream at the city locations (e.g. BL09, BL10). The increasing influence of the urban roughness on the flow field above the canopy is reflected in the fact that the size of the energy-containing eddies, measured by the frequency location of the energy peaks, is gradually decreasing. A similarly good agreement between both data sets is also seen below roof level at $0.5 H$ (not shown).

The bottom row of Fig. 8 shows co-spectra, $\mathrm{Co}_{u w}$, of the streamwise and vertical velocity fluctuations. Compared to the auto-spectra the inertial-subrange slopes of the co-spectra are much steeper with a $-4 / 3$ power-law decay $[63,25]$. This indicates the importance of large eddies for the vertical turbulent momentum exchange $\overline{u^{\prime} w^{\prime}}$ in the urban roughness sublayer. At all sites, the roll-off of the LES spectra is considerably faster, in agreement with the findings for the auto-spectra. Overall the flux-dominating frequency ranges in the LES agree very well with the wind tunnel. Even complex features like the double-peak pattern observed at location BL10 are remarkably well captured. In both data sets, the spectral maxima are shifting towards higher frequencies from the river site BL04 (not shown) to the downstream street canyon sites. This shows that the LES is captures the increasing influence of the urban environment on the flow and the importance of building-induced turbulence for vertical turbulent mixing.

\section{Flow structures}

The previous analyses are classic ways to infer information about the structure of turbulence, characteristic scales of dominant eddies and associated contributions to the variance of the flow field. In the next and final level of the validation study we now extend this analysis by investigating the dynamics of eddy structures by means of conditional resampling of the vertical momentum flux and joint time-frequency analysis.

\subsection{Quadrant analysis}

The comparison of the $\left(u^{\prime}, w^{\prime}\right)$ co-spectra showed that the LES provides a realistic picture of the average flux contributions from different eddy classes. In the following, the structure of the instantaneous vertical turbulent momentum flux $\overline{u^{\prime} w^{\prime}}$ is examined with quadrant analysis. In a first step, the flux fractions defined in Eq. (4) are related to the associated joint probability density function (JPDF) of $u^{\prime} / U_{r e f}$ and $w^{\prime} / U_{r e f}$. This approach provides a 2D extension of the analysis of velocity frequency distributions presented in part I.

Fig. 9 shows comparisons of JPDFs at two example locations in a height of $z=1.3 \mathrm{H}$ : site BL07, located just downstream of the river upstream of the city core, and the downtown site BL10 at a complex intersection. Qualitatively, the overall shapes and extents of the joint PDFs in the $\left(u^{\prime} / U_{r e f}, w^{\prime} / U_{r e f}\right)$ plane agree well. At both locations, the semi-major axes of the ellipses proceed through the $Q_{2}$ and $Q_{4}$ quadrants, indicating that the largest instantaneous flux amplitudes are associated with ejection and sweep episodes. At site BL07 both joint PDFs are fairly symmetrical about the semi-major and semi-minor axes, with 

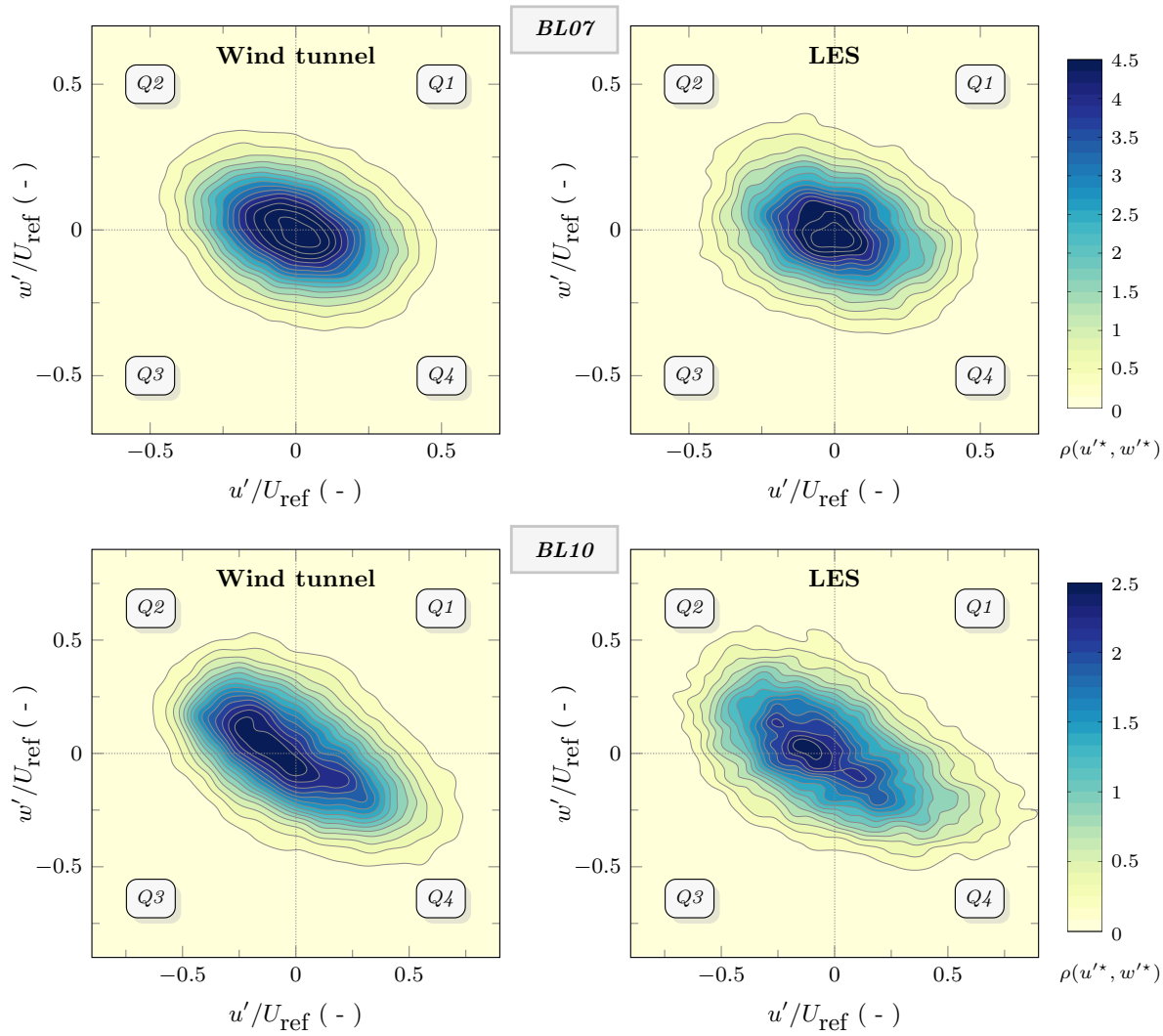

Fig. 9: Joint probability density functions of the streamwise and vertical velocity fluctuations in the wind tunnel (left) and the LES (right) for two comparison sites at $z=1.3 H(45.5 \mathrm{~m})$. Note that $u^{\prime \star}=u^{\prime} / U_{\text {ref }}$ and $w^{\prime \star}=w^{\prime} / U_{\text {ref }}$ in the legend. See insets in Fig. 10 for the locations of sites BL07 and BL10.

the peaks being centred at low amplitudes of $u^{\prime} / U_{\text {ref }}$ and $w^{\prime} / U_{\text {ref }}$. This picture changes significantly when investigating the flow above the city centre, which is increasingly affected by the change in the underlying surface roughness. At BL10 the LES and the wind tunnel both show an overall increase of high-amplitude fluxes in the $Q_{2}$ and $Q_{4}$ quadrants. However, while the distribution peaks both shift away from the centre into the low-amplitude region of the ejection quadrant $\left(Q_{2}\right)$, strong downward motions (sweeps, $\left.Q_{4}\right)$ at this location are occurring more often than their ejection counterparts. The larger variability observed in the LES probability contour lines is associated both with the eddy-scale truncation in the simulation and with the shorter duration of the LES signals compared to the experiment.

\subsubsection{Flux fraction profiles}

Vertical profiles of flux fractions $S_{i}$, local sweep-ejection differences $\delta S_{4,2}$ and of the exuberance parameter Ex above the UCL are shown in Fig. 10 at three sites. Since ejection and sweep episodes are associated with coherent turbulence structures primarily associated with 

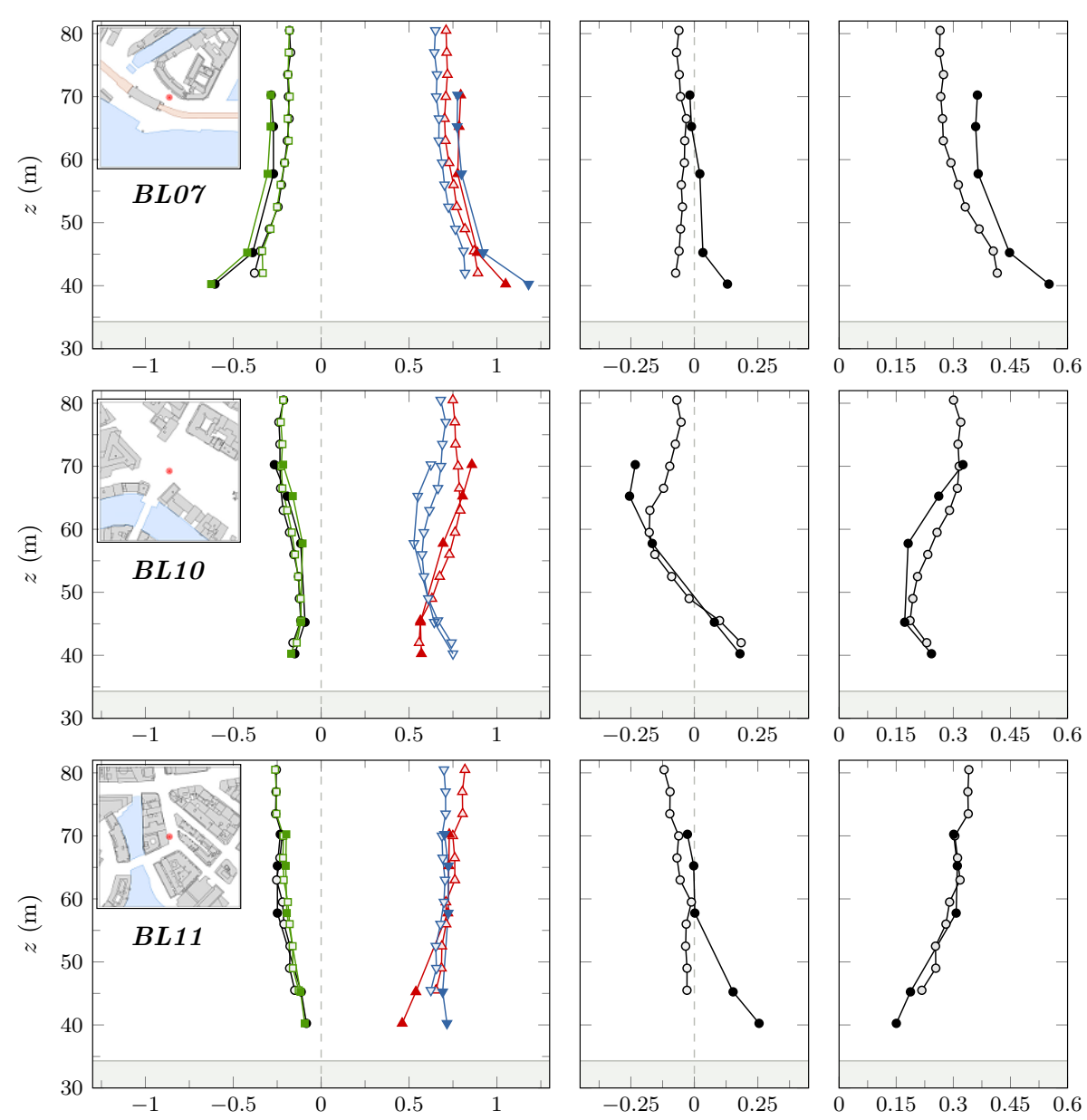

\begin{tabular}{ll|}
$\multimap-S_{1}$ Wind tunnel & $-S_{1}$ LES \\
$-\square-S_{2}$ Wind tunnel & $-S_{2}$ LES \\
$\multimap-S_{3}$ Wind tunnel & $-S_{3}$ LES \\
$\square-S_{4}$ Wind tunnel & $-S_{4}$ LES
\end{tabular}

$\delta S_{4,2}(-)$

$|E x|(-)$

$\multimap$ Wind tunnel $\longrightarrow$ LES

Fig. 10: Comparison of vertical profiles of wind tunnel (open symbols) and LES (filled symbols) flux fractions $S_{i}$ (left), local differences between sweeps and ejections $\delta S_{4,2}$ (centre) and magnitudes of the exuberance $|E x|$ (right) above the UCL. The grey shading indicates heights lower than $H$.

large-scale eddies [10], turbulence-resolving time-dependent simulations should be able to resolve their general characteristics.

At all sites ejection and sweep episodes clearly dominate the flow compared to countergradient fluxes, which is in agreement with other studies on surface-layer turbulence and flow characteristics within and above urban canopies, e.g. [41,44,37,17,10]. Overall, the LES captures the flux magnitudes associated with the respective quadrants. The qualitative 
response of the momentum exchange to the local roughness characteristics strongly resembles the laboratory observations. Slight differences in trends are found close to roof level, where the LES predicts a dominance of downward sweep motions $\left(Q_{4}\right)$ at all sites, while the laboratory flow shows a slight prevalence of ejections with the exception of location BL10. Higher up in the roughness sublayer, both the LES and the experiments show an ejection prevalence. While several of the studies cited above have documented a dominance of sweeps within and just above the canopy layer, this prevalence clearly is connected to the morphological characteristics of the analysis site and to the local flow structure. Especially in strongly heterogeneous canopies, like in this study, inferring general conclusions from local analyses is difficult.

At the most upstream site BL07 the largest quantitative offsets between the wind tunnel and LES $S_{i}$ profiles are observed, while qualitatively the height-dependent characteristics overall are well reproduced. Compared to locations further downstream here the LES and wind-tunnel flows are characterised by a larger proportion of counter-gradient fluxes at lower elevations. However, the exchange efficiency gradually increases with height as seen in the exuberance profiles $|E x(z)|$. The smaller the exuberance magnitude, the more efficient the vertical turbulent momentum exchange through ejections and sweeps. The picture is different at the downtown sites BL10 and BL11, where the roughness-layer flow now is increasingly affected by three-dimensional building-induced mixing. The vertical momentum exchange is more efficient close to roof level (dominance of $S_{2}+S_{4}$ over $S_{1}+S_{3}$ ) and becomes less efficient at higher elevations. At the intersection site BL10 the qualitative and quantitative agreement is remarkably good. In the experiment and the LES the lowest comparison points are associated with a dominance of sweeps, while upward ejections are dominant further away from the canopy. The height profiles of $\delta S_{4,2}$ exhibit strong gradients in a region between approximately $1.3 H$ to $2 H$, reflecting an enhanced turbulent exchange between the flow field influenced by UCL turbulence and the upper-level flow. The largest magnitudes of Ex up to values of 0.6 are found at the lowest comparison heights at BL07. In contrast to that, at BL10 and BL11 the momentum exchange efficiency above the UCL is stronger: a change that is captured by the LES. The height ranges determined for the most efficient vertical momentum exchange are similar to those reported by Christen et al. [10] $(1.0<z / H<1.25)$ from analyses of field measurements in a street canyon. In both the LES and the experiment, $|E x|$ converges to a nearly constant value of about 0.3 above $2 H$, indicating a Gaussian distribution of the JPDFs in the $\left(u^{\prime} / U_{r e f}, w^{\prime} / U_{r e f}\right)$ plane, again in agreement with field observations [10].

\subsubsection{Extreme events}

The above results show that the LES provides a realistic picture of time-dependent eddy dynamics in terms of instantaneous flux contributions from episodes of downward motions of air from the roughness sublayer towards the canopy and upward bursts of low-momentum fluid at different locations throughout the city. Such events often occur intermittently with large amplitudes of the instantaneous $u^{\prime} w^{\prime}$ fluxes as illustrated by the JPDFs (Fig. 9) and play an important role for canopy-layer ventilation or local detrainment and re-entrainment characteristics in street canyons. The occurrence of such strong ejection and sweep episodes can be related to the propagation of large-scale coherent eddy structures at the top of the canopy layer and to the contributions of building induced vortex shedding [27,11].

In order to quantify and compare the relative importance of large amplitude contributions to the flux fractions, a threshold parameter (hole size) $H_{c}$ is introduced in a next step 

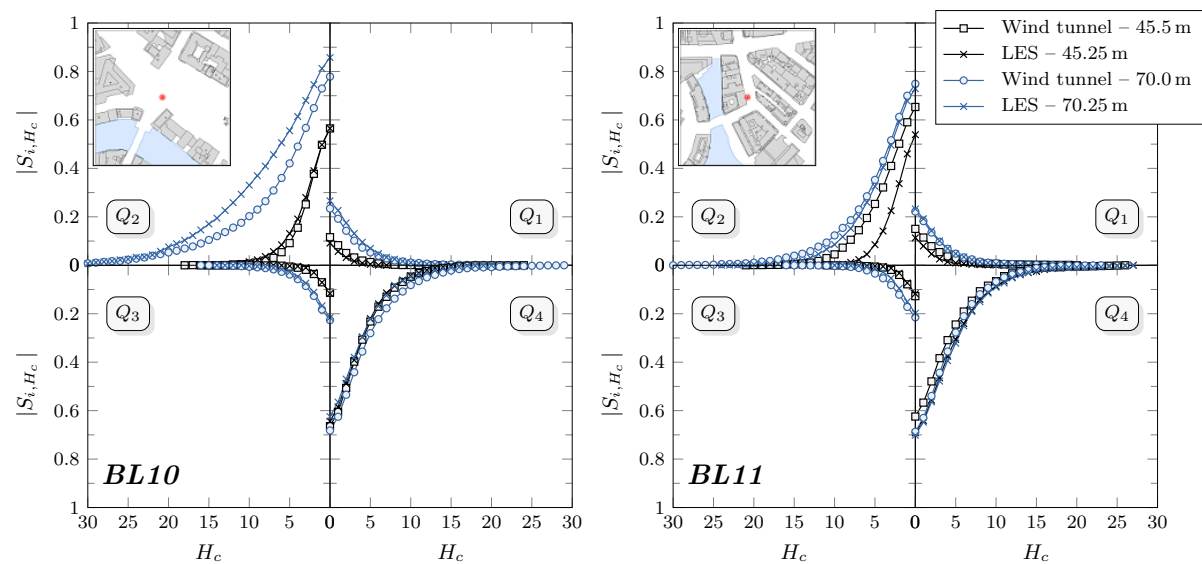

Fig. 11: The four flux fractions, $\left|S_{i, H_{c}}\right|$, as a function of hole size, $H_{c}$, in the wind tunnel (dots) and LES (crosses) at two comparison sites in heights of $1.3 H(45.5 \mathrm{~m})$ and $2 H(70 \mathrm{~m})$.

as a further constraint on the conditional averaging (see Sect. 2.2). Low-amplitude contributions to the total flux are successively filtered out such that the comparatively rare but strong remaining contributions to the momentum transport can be studied.

Results of this analysis are shown in Fig. 11 for two heights within the roughness sublayer: $1.3 H(45.5 \mathrm{~m})$ and $2 H(70.25 \mathrm{~m})$ at the city-centre sites BL10 and BL11. Depicted are flux fraction magnitudes, $\left|S_{i, H_{c}}\right|$, as a function of hole size, $H_{c}$, for which we used a maximum value of 30 to cover the entire event space. The overall agreement of the hole-size dependent flux fractions computed from wind tunnel and the LES velocity time series is very high with regard to their qualitative and quantitative evolution. At both locations the dominance of ejection and sweep contributions $\left(Q_{2}, Q_{4}\right)$ over the interaction quadrants $\left(Q_{1}\right.$, $Q_{3}$ ) is preserved as the hole size is increased. Flux contributions from the counter-gradient $Q_{1}$ and $Q_{3}$ quadrants rapidly drop off as $H_{c}$ is increased, showing that occurrences of strong flux episodes in these quadrants are very unlikely at the investigated sites. The only significant difference between the simulation and the experiment occurs at site BL10 in the $Q_{2}$ quadrant at $70.25 \mathrm{~m}$. Here the LES predicts larger contributions from high-amplitude fluxes than evident in the experiment. The corresponding JPDFs (Fig. 9) indicate that this is likely connected to the occurrence of slightly larger negative streamwise velocity fluctuations. In the second analysis height at the same location, however, a very good qualitative and quantitative agreement of the flux-fraction evolution in all four quadrants is found. Here the prevalence of ejection motions in the upper parts of the roughness sublayer is accompanied by significant instantaneous turbulent flux episodes at large $H_{c}$. This is also the case at location BL11, where at both heights the behaviour of $\left|S_{i, H_{c}}\right|$ in the experiment and the simulation is quantitatively very similar.

\subsection{Wavelet analysis}

In the final level of the validation study, we analyse joint time-frequency information contained in the wavelet coefficients $W_{n}(s)$ derived from velocity fluctuations as a time dependent extension of classic Fourier analysis. In order to study the turbulent flow with regard 
to the occurrence of certain eddy structures, the wavelet coefficients are analysed in terms of frequency distributions of the wind tunnel and LES data. For this purpose, the wavelet transform according to Eq. (6) is conducted using the Morlet wavelet (Fig. 4) for wavelet scales corresponding to frequencies in the energy-containing spectral range, resulting in frequency-dependent time series of wavelet coefficients.

Fig. 12 shows frequency distributions of experimental and LES wavelet coefficients corresponding to extraction frequencies of $f^{\star}=f z / \bar{U}=0.25,0.75$ and 1.0. These frequencies are all within the spectral peak range associated with eddies involved in turbulence production (see 1D spectra in Fig. 8). It was ensured that the fast roll-off of the LES spectra had not yet started at these frequencies, so that the information contained in the wavelet coefficients still corresponds to directly resolved scales. At these frequencies the local wavelet spectra are neither affected by aliasing at the highest frequencies nor by end effects arising from the analysis of signal portions at the beginning or end of the time series. The coefficients shown in Fig. 12 were obtained from streamwise velocity fluctuations in a height of $17.5 \mathrm{~m}$ $(\sim 0.5 H)$ at four sites corresponding to different urban settings. The time-dependent wavelet coefficients $W_{n}\left(f^{\star}\right)$ were normalised by the respective standard deviations of the coefficient time series, $\sigma_{W}$. Results are presented using semi-logarithmic axes since we are particularly interested in the tails of the distributions, which contain information about rare, intermittent events in the flow that leave a distinct footprint in the amplitudes of the wavelet coefficients. For a quantification of the level of agreement between the experimental and LES frequency distributions the kurtosis $\beta$ of the samples are derived and displayed. In order to determine deviations from a normal distribution the corresponding Gaussian curves are shown.

A common feature evident at all sites is that the wavelet coefficients most often exhibit small negative or positive amplitudes. The behaviour in the tails, on the other hand, is different at every site. This reflects differences in the local flow structure, but also shows a clear dependency on the frequency at which the coefficients are analysed. The smallest deviations from a normal distribution are found at the lowest frequency selected. For $f^{\star}=0.75$ and higher, the distributions feature heavier tails, reflecting an enhanced and intermittent activity in the flow associated with rare events and velocity bursts [16]. Deviations from the normal distribution are quantified by the respective values of $\beta$, which partially show significant offsets from the Gaussian reference $(\beta=3)$. In particular, the coefficient distributions tend to be more leptokurtic, i.e. they exhibit higher peaks and heavier tails than the normal distribution. This feature is seen in the experiment and the LES and similar frequency-dependent distribution characteristics can be determined at different comparison locations.

At all sites the LES predictions are qualitatively and quantitatively in good agreement with the wind-tunnel experiment. At the intersection position BL10, for example, both the wind-tunnel and the LES wavelet coefficient distributions exhibit extended exponential tails at the two highest extraction frequencies, recognisable as a linear decay in this semi-logarithmic representation. This feature illustrates the influence of the increased surface roughness on the spatial scales of dominant flow structures. Quadrant analysis showed that the location above the intersection is characterised by strong and intermittent vertical momentum exchange, dominated by sweep events. Similar tail behaviour is also detected at other comparison locations, notably at the river location BL04 $\left(f^{\star}=1.0\right)$. The kurtosis values indicate that the LES distributions have a tendency to be slightly more leptokurtic than the reference at $f^{\star}=1.0$, i.e. high amplitude oscillations in the wavelet coefficients are occurring more frequently than in the reference experiment. This could be a result of the proximity to the steep drop-off observed in the energy spectra. The increased level of intermittency could be an indication for the increased influence of the grid cut-off in the transition region between resolved and unresolved turbulence. 

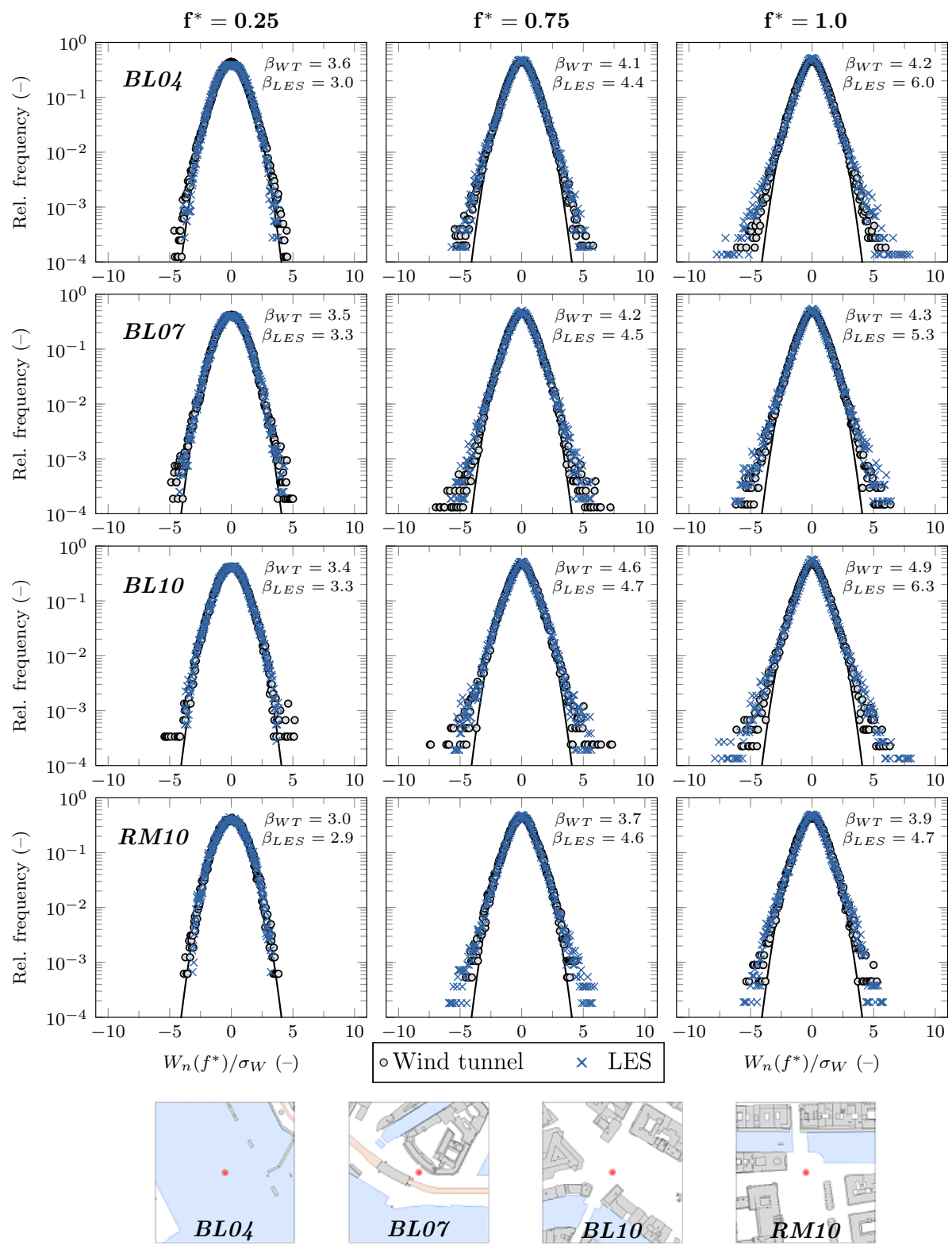

Fig. 12: Comparison of wind tunnel (dots) and LES (crosses) frequency distributions of Morlet wavelet coefficients derived from streamwise velocity fluctuations at four locations in a height of $0.5 H(17.5 \mathrm{~m})$. The distributions correspond to scaled frequencies of $f^{\star}=$ $f z / \bar{U}=0.25$ (left), 0.75 (centre) and 1.0 (right). The black lines show the corresponding Gaussian distributions. 


\section{Discussion and conclusions}

\subsection{Evaluation of the Hamburg test case}

The Hamburg validation test case showed that the quality of LES can be assessed in detail by not only focusing on comparisons of low-order statistics, but by taking into account the time-dependent nature of the problem. Following the multi-level validation concept proposed in part I we established that the tested LES code, FAST3D-CT, provides a realistic representation of mean flow and turbulence statistics in the urban roughness sublayer. This assessment was further substantiated by a direct comparison of time series in terms of frequency distributions, showing that the LES accurately reproduces complex geometryinduced flow patterns, e.g. flow-switching events reflected in bimodal velocity histograms. In part II presented here we have extended the analysis by examining the underlying flow structure in detail with regard to time-dependent flow statistics and flow features associated with energy and flux-dominating eddies that are directly resolved by the LES.

\subsubsection{Eddy scale and flow pattern analysis}

Compared to small-scale turbulence structures, energy and flux-dominating large-scale vortices occur less frequently. The representativity of associated statistical measures thus is strongly coupled to the duration of the signal, i.e. to the measurement or simulation time. Computational costs and computing time restrictions often result in the fact that there is a significant difference in LES signal lengths compared to the reference data, as was the case in our example study. While in this case the inherent uncertainty of low-order statistics obtained from the longer wind tunnel and shorter LES time series $\left(T_{\exp }=16.5 \mathrm{~h}\right.$ and $T_{l e s}=6.5 \mathrm{~h}$ ) is negligible compared to the experimental reproducibility, this may not be true for more sensitive parameters. An example is $S_{i, H_{c}}$, which measures the relevance of extreme flux episodes associated with large, infrequently occurring eddies. The magnitude of this effect can for example be assessed based on the longer experimental data record. Repeating the hole-size analysis of Sect. 4.1.2 with a wind-tunnel time series that is shorter by a factor of $T_{\exp } / T_{\text {les }}=2.5$ showed non-negligible differences in the flux fractions of the $Q_{2,4}$ quadrants that are associated with coherent eddies. The larger the number of energydominating structures that have passed the sensor, the more robust are statistics associated with this eddy class. This has to be kept in mind when evaluating the agreement between LES and experiment.

Eddy statistics Since reference data are still predominantly available in terms of single-point measurements, as was also the case in the test study, the focus should be on comparisons of dominant turbulence time scales and temporal autocorrelations to learn about the structure of the flow. In general, this can be extended into spatial correlation analyses given reference data of sufficient spatial resolution. For the model tested here, we found that the height dependence of integral time scales of the three velocity components overall is well reproduced in the simulation. Given the complexity of the canopy-layer flow field and the general sensitivity of measures based on correlations, this underlines the general suitability of the tested code for urban aerodynamics simulations.

Analysing spectral energy densities allows the evaluation of contributions from eddies of different size to the local flow variance. With LES it is of particular interest to investigate up to what frequency range turbulence structures are directly resolved, which can be readily determined by the characteristic fast roll-off of the spectra as a result of the grid truncation. 
In the Hamburg LES, the roll-off occurred in the transition region between the turbulence production range and the inertial subrange. Within the UCL, a scale decrease of the production range eddies is clearly reflected in the wind-tunnel spectra as a shift of the energy peaks towards higher frequencies. Given this eddy size reduction, the uniform grid resolution of $2.5 \mathrm{~m}$ in combination with the implicit dissipation scheme causes the LES to be at the verge of being a "very large-eddy simulation" within the UCL. Despite this resolution limitation, at the majority of comparison sites the spectral shapes in the energy-dominating frequency range agree well with the experimental targets. This applies to frequency ranges associated with the spectral peak region and to the distribution of energy among the largest eddies in the flow, demonstrating that the LES accurately resolves turbulence that dominate the flow in terms of turbulence kinetic energy and turbulent mixing.

Eddy structure and flow dynamics Within the atmospheric surface layer, turbulence is highly three-dimensional, particularly so within the urban canopy layer. Whether eddy-resolving simulation techniques are resolving the structure of turbulence in a realistic way can be analysed by means of structure identification methods, e.g. quadrant analysis, as done for the Hamburg test case. We showed that the efficiency of vertical momentum exchange, which is a quantity of interest for street-canyon ventilation or vertical detrainment, can be used as a quality control measure for the simulation. In the test case, for example, the LES produced momentum flux characteristics that are in agreement with the reference case regarding the local dominance of upward or downward motions associated with coherent eddy structures (gradient-type motions). Carrying out these comparisons at structurally different flow locations allows the study of changes in the characteristics of flux events in response to the underlying roughness. By introducing a hole-size constraint to the conditional re-sampling process, the occurrence of flux contributions linked to infrequent, high-amplitude events in the flow was evaluated. The good agreement of the $H_{c}$-dependent flux fractions shows that the LES realistically represents the local dominance of downward sweeps of highmomentum fluid towards the UCL and upward ejections of low-momentum fluid into the upper parts of the roughness sublayer.

Extending the classic Fourier analysis, the time-dependent flow structure can be compared in a joint time-frequency framework. Scale-dependent analyses of the turbulent flow field based on wavelet transform methods offer strong potential here, and in the Hamburg test case revealed a structural agreement of the time-dependent experimental and LES flows. The comparison of frequency distributions of wavelet coefficients extracted at energetically dominant frequencies reveal occurrence characteristics of rare but energetically significant turbulence episodes. Depending on the extraction frequency and the comparison location, the wavelet coefficient PDFs can feature heavy tails, which is an indication for an increased level of intermittency in the flow. By comparing the kurtosis values associated with the distributions the level of agreement can be quantified.

\subsubsection{Model accuracy}

Applying the proposed validation strategy showed that FAST3D-CT provides realistic and reliable information about urban turbulence with regard to geometry-induced mean and instantaneous flow features. Despite the overall strong performance of FAST3D-CT some systematic discrepancies were identified. In part I we discussed how the grid resolution of $2.5 \mathrm{~m}$ in combination with the computational representation of buildings by simple grid masking negatively affected the comparison, particularly in narrow street canyons. Spectral analyses performed in part II revealed how the grid resolution also affected the resolution 
potential of flow structures in the implicit LES. Although a grid-resolution of $2.5 \mathrm{~m}$ should make it possible to resolve at least one frequency decade of inertial subrange turbulence, the FCT-scheme handling the numerical dissipation in the model in its current configuration seemed to contribute to an enhanced energy loss, identifiable in the turbulence spectra. In general it can be expected that this also has an effect on mean flow and turbulence statistics, adding to the other sources of discrepancies identified before. Within the UCL, FAST3D-CT in this study only fully resolved eddies in the production range, which is characteristic for a very large-eddy simulation. The grid resolution also affected the overall comparability of the data due to spatial offsets between comparison locations in the experiment and the LES. Seemingly marginal height differences in the order of $0.25 \mathrm{~m}$ can have a significant influence on the results in regions of strong flow gradients. The same is true for spatial offsets in the $(x, y)$ plane in strongly heterogeneous flow situations or near building walls. Re-running the Hamburg case with a more detailed representation of buildings, e.g. based on unstructured meshes or immersed boundary methods, would certainly lead to a better understanding of some of the discrepancies determined here. It can be expected that there will be improvements particularly at comparison points located in narrow streets. The same can be expected of refinements of the grid resolution in connection with the FCT-scheme, and improvements of the inflow modelling. In order to disentangle these overlapping sources of inaccuracy and to determine the effects of a better reproduction of low-frequency inertial range eddies, the resolution would need to be increased to $1 \mathrm{~m}$ or below. However, these improvements inevitably lead to higher computational costs. For example, doubling the grid resolution will result in a sixteen-fold increase in run time. Alternatively, the simulation could be re-run in a much smaller domain, making the increase in grid resolution affordable, while possibly also being able to explore more accurate buildings representation techniques.

\subsection{Conclusions and outlook}

The work presented here was motivated by the lack of proportion between the increasing use of eddy-resolving CFD methods like LES for micro-meteorological and environmental fluid mechanics applications and the level of scrutiny that is commonly applied to validate the simulation results. Based on the example of highly complex urban boundary-layer flow we showed that through a rigorous validation against qualified reference data based on model-specific tests, the suitability of time-dependent, turbulence-resolving simulations for their intended use can be documented, and the bounds of uncertainty in the results can be quantified. With well-established signal analysis methods and by means of suitable and quality-controlled reference data, a high level of detail can be incorporated into the validation of LES. The information gained by far exceeds what can be learned based on the traditional approach of comparing mean flow and turbulence statistics. By applying the sequence of analysis methods to the Hamburg flow simulation we were able to validate, for example, the representation of

- Velocity fluctuations in response to local flow structure

- Time scales associated with dominant turbulent eddies

- Distribution of energy among flow structures of different size

- Turbulent exchange efficiency associated with different eddy structures

- Contribution of extreme events to local turbulent exchange characteristics

- Flow intermittency captured in time-dependent energy spectra.

While the validation method was tested based on the specific case of urban turbulence, it can also be applied to validate other types of eddy-resolving boundary-layer simulations, 
for example flow within and above plant canopies or above a uniform roughness (e.g. only prescribed through the roughness length). Similar validation concepts based on time-series analyses can also be applied to validate simulations of scalar dispersion or heat exchange. The methods can also be applied to other types of turbulence-resolving simulations to study local-scale problems like detached eddy simulation or direct numerical simulations, but also to high-resolution meteorological models $(O(10-100 \mathrm{~m}))$ applied to study problems in which length-scales of energy-dominating eddies scale with the boundary-layer depth.

The study showed that experiments in boundary-layer wind tunnels with full control over mean inflow and boundary conditions, flexibility in the geometric design of the test case and measurement repeatability offer great potential for model validation. However, wind-tunnel experiments themselves are models with strong geometric and physical abstractions. The potential, for example, to realistically model effects like differential heating on surfaces, anthropogenic heating, evapo-transpiration from vegetation, strong convection or stable stratification in wind tunnels is limited. Therefore, ideally laboratory studies are accompanied by full-scale field experiments and vice versa. While only the latter can capture the full complexity of natural atmospheric flows, the former allows the systematic study of relevant processes in isolation. Urban studies for which field and wind-tunnel data are available and in the past were used for comprehensive model validation include for example the DAPPLE experiment in central London [62], the MUST experiment in the Utahn desert $[5,47]$ or the Joint Urban 2003 campaign in Oklahoma City [24].

LES is increasingly applied to real-life problems of practical concern and quality assurance is becoming more and more crucial. Community-wide activities are now needed in order to streamline model validation efforts. Micro-meteorology and wind engineering communities have demonstrated before that multi-national, multi-institutional activities for the harmonisation of validation approaches for flow in urban environments are feasible. The COST Actions $732[7,48,21]$ and ES1006 [58] are encouraging examples of how exchange between experimentalists and modellers can result in broadly accepted model quality standards, best-practice simulation protocols and validation guidelines for flow and dispersion models applied to problems on the urban micro-scale. Similar activities need to be pursued for LES, involving model developers and users as well as field and laboratory experimentalists. For micro-meteorological and environmental fluid mechanics applications in the nearsurface boundary layer, the joint formulation of validation guidelines need to stress the role of advanced turbulence analysis methods and flow structure recognition techniques, and the need for standards regarding quality and quantity requirements for reference data.

Acknowledgements The numerical simulations with the LES code FAST3D-CT were carried out at the Laboratories for Computational Physics and Fluid Dynamics of the U.S. Naval Research Laboratory in Washington D.C., USA. The authors wish to express their thanks to Jay Boris, Mi-Young Obenschain and other collaborators there. Further thanks is given to colleagues at the Environmental Wind Tunnel Laboratory at the University of Hamburg. Financial funding by the German Federal Office of Civil Protection and Disaster Assistance as well as by the Ministry of the Interior of the City of Hamburg within the "Hamburg Pilot Project" is gratefully acknowledged (BBK research contract no. BBK III.1-413-10-364). Parts of the wind-tunnel model construction were financially supported by the KlimaCampus at the University of Hamburg.

\section{References}

1. Addison, P.S.: The illustrated wavelet transform handbook: Introductory theory and applications in science, engineering, medicine and finance. Institute of Physics Publishing, London (2002)

2. Adrian, R.J.: Hairpin vortex organization in wall turbulence. Phys Fluids 19, 041,301 (2007) 
3. Basu, S., Vinuesa, J.F., Swift, A.: Dynamic les modeling of a diurnal cycle. J Appl Meteorol Climatol 47, 1156-1174 (2008)

4. Beare, R.J., Macvean, M.K., Holtslag, A.A.M., Cuxart, J., Esau, I., Golaz, J.C., Jimenez, M.A., Khairoutdinov, M., Kosovic, B., Lund, D.L.T.S., Lundquist, J.K., McCabe, A., Moene, A.F., Noh, Y., Raasch, S., Sullivan, P.: An intercomparison of large-eddy simulations of the stable boundary layer. Bound-Lay Meteorol 118, 247-272 (2006)

5. Biltoft, C.A.: Customer report for MOck Urban Setting Test (MUST). Tech. Rep. WDTC-FR-01-121, West Desert Test Center, U.S. Army Dugway Proving Ground, Dugway (UT), USA (2001)

6. Boppana, V.B.L., Xie, Z.T., Castro, I.P.: Large-eddy simulation of dispersion from surface sources in arrays of obstacles. Bound-Lay Meteorol 135, 433-454 (2010)

7. Britter, R., Schatzmann, M. (eds.): Model evaluation guidance and protocol document. COST Action 732. University of Hamburg, Germany (2007b)

8. Chlond, A., Böhringer, O., Auerswald, T., Müller, F.: The effect of soil moisture and atmospheric conditions on the development of shallow cumulus convection: A coupled large-eddy simulation land surface model study. Meteorol Z 23(5), 491-510 (2014)

9. Chow, F.K., Weigel, A.P., Street, R.L., Rotach, M.W., Xue, M.: High-resolution large-eddy simulations of flow in a steep alpine valley. Part I: Methodology, verification, and sensitivity experiments. J Appl Meteorol Climatol 45, 63-86 (2006)

10. Christen, A., van Gorsel, E., Vogt, R.: Coherent structures in urban roughness sublayer turbulence. Int J Climatol 27, 1955-1968 (2007)

11. Coceal, O., Dobre, A., Thomas, T.G.: Unsteady dynamics and organized structures from DNS over an idealized building canopy. Int J Climatol 27, 1943-1953 (2007)

12. Conzemius, R., Fedorovich, E.: Bulk models of the sheared convective boundary layer: Evaluation through large eddy simulations. J Atmos Sci 64, 786-807 (2007)

13. Cooley, J.W., Tukey, J.W.: An algorithm for the machine calculation of complex Fourier series. Math Comput 19, 297-301 (1965)

14. Deardorff, J.W.: A numerical study of three-dimensional turbulent channel flow at large Reynolds numbers. J Fluid Mech 41, 453-480 (1970)

15. Farge, M.: Wavelet transforms and their application to turbulence. Annu Rev Fluid Mech 24, 395-457 (1992)

16. Farge, M., Schneider, K., Pannekoucke, O., Nguyen van yen, R.: Multiscale representations. In: H.J.S. Fernando (ed.) Handbook of Environmental Fluid Dynamics, vol. II: Systems, Pollution, Modeling, and Measurements. CRC Press (2012)

17. Feddersen, B.: Wind tunnel modelling of turbulence and dispersion above tall and highly dense urban roughness. Ph.D. thesis, Swiss Federal Institute of Technology (2005). Diss. ETH No. 15934

18. Feigenwinter, C., Vogt, R.: Detection and analysis of coherent structures in urban turbulence. Theor Appl Climatol 81, 219-230 (2005)

19. Finnigan, J.J.: Turbulence in plant canopies. Annu Rev Fluid Mech 32, 519-571 (2000)

20. Fischer, R.: Entwicklung eines problemorientierten Software-Pakets zur automatisierten Aufbereitung, Analyse und Dokumentation von im Windkanal produzierten Daten zur LES-Validierung. Ph.D. thesis, University of Hamburg (2011). In German

21. Franke, J., Hellsten, A., Schlünzen, K.H., Carissimo, B.: The COST 732 Best Practice Guideline for CFD simulation of flows in the urban environment: a summary. Int J Environ Pollut 44, 419-427 (2011)

22. Grossmann, A., Morlet, J.: Decomposition of Hardy functions into square integrable wavelets of constant shape. SIAM J Math Anal 15, 723-736 (1984)

23. Grossmann, A., Morlet, J., Paul, T.: Transforms associated to square integrable group representations I: General results. J Math Anal 26, 2473-2479 (1985)

24. Harms, F., Leitl, B., Schatzmann, M., Patnaik, G.: Validating LES-based flow and dispersion models. J Wind Eng Ind Aerod 99, 289-295 (2011)

25. Kaimal, J.C., Wyngaard, J.C., Izumi, Y., Coté, O.R.: Spectral characteristics of surface-layer turbulence. Q J Roy Meteor Soc 98, 563-589 (1972)

26. Kaiser, G.: A Friendly Guide to Wavelets, first edn. Birkhäuser Boston (1994)

27. Kanda, M., Moriwaki, R., Kasamatsu, F.: Large-eddy simulation of turbulent organized structures within and above explicitly resolved cube arrays. Bound-Lay Meteorol 112, 343-368 (2004)

28. Letzel, M.O.: Urban large-eddy simulation (LES), Advanced computational fluid dynamics for urban climatic maps. In: E. Ng, C. Ren (eds.) The Urban Climatic Map: A Methodology for Sustainable Urban Planning, chap. 32, pp. 421-428. Routledge, New York (2015)

29. Letzel, M.O., Raasch, S.: Large eddy simulation of thermally induced oscillations in the convective boundary layer. J Atmos Sci 60, 2328-2341 (2003)

30. Lilly, D.K.: The representation of small-scale turbulence in numerical simulation experiments. In: H.H. Goldstine (ed.) Proceedings of the IBM Scientific Computing Symposium on Environmental Sciences, pp. 195-210. Yorktown Height, New York (1967) 
31. Lumley, J.L., Panofsky, H.A.: The Structure of Atmospheric Turbulence. Interscience Publishers, New York (1964)

32. Meneveau, C.: Analysis of turbulence in the orthonormal wavelet representation. J Fluid Mech 232 , 469-520 (1991)

33. Michioka, T., Chow, F.K.: High-resolution large-eddy simulation of scalar transport in atmospheric boundary layer flow over complex terrain. J Appl Meteorol Climatol 47, 3150-3169 (2008)

34. Morlet, J.: Sampling theory and wave propagation. In: Proceedings of the 51st Annual International Meeting of the Society of Exploration Geophysicists. Los Angeles (CA), USA (1981)

35. Nieuwstadt, F.T.M., Mason, P.J., Moeng, C.H., Schumann, U.: Large-eddy simulation of the convective boundary layer: A comparison of four computer codes. In: F. Durst, B.E. Launder, R. Friedrich, F.W. Schmidt (eds.) Turbulent Shear Flows 8, pp. 343-367. Springer (1993)

36. Nobach, H., Tropea, C., Cordier, L., Bonnet, J.P., Delville, J., Lewalle, J., Farge, M., Schneider, K., Adrian, R.J.: Review of some fundamentals of data processing. In: C. Tropea, A.L. Yarin, J.F. Foss (eds.) Springer Handbook of Experimental Fluid Mechanics, Part D: Analysis and Post-Processing of Data. Springer (2007)

37. Oikawa, S., Meng, Y.: Turbulence characteristics and organized motion in a suburban roughness sublayer. Bound-Lay Meteorol 74, 289-312 (1995)

38. O'Neill, P.L., Nicolaides, D., Honnery, D., Soria, J.: Autocorrelation functions and the determination of integral length with reference to experimental and numerical data. In: Proceedings of the 15th Australasian Fluid Mechanics Conference. The University of Sydney, Sydney, Australia (2004)

39. Patnaik, G., Boris, J.P., Grinstein, F.F., Iselin, J.P., Hertwig, D.: Large scale urban simulations with FCT. In: D. Kuzmin, R. Löhner, S. Turek (eds.) Flux-Corrected Transport: Principles, Algorithms, and Applications, Scientific Computing, second edn., pp. 91-117. Springer (2012)

40. Patnaik, G., Grinstein, F.F., Boris, J.P., Young, T.R., Parmhed, O.: Large-scale urban simulations. In: F.F. Grinstein, L.G. Margolin, W.J. Rider (eds.) Implicit Large Eddy Simulation: Computing Turbulent Fluid Dynamics. Cambridge University Press (2007)

41. Raupach, M.R.: Conditional statistics of Reynolds stress in rough-wall and smooth-wall turbulent boundary layers. J Fluid Mech 108, 363-382 (1981)

42. Raupach, M.R., Thom, A.S.: Turbulence in and above plant canopies. Ann Rev Fluid Mech 13, 97-129 (1981)

43. Robinson, S.K.: Coherent motions in the turbulent boundary layer. Annu Rev Fluid Mech 23, 601-639 (1991)

44. Rotach, M.W.: Turbulence close to a rough urban surface - Part I: Reynolds stresses. Bound-Lay Meteorol 65, 1-28 (1993)

45. Rotach, M.W.: Profiles of turbulence statistics in and above an urban street canyon. Atmos Environ 29, 1473-1486 (1995)

46. Roth, M.: Review of atmospheric turbulence over cities. Q J Roy Meteor Soc 126, 941-990 (2000)

47. Sabatino, S.D., Buccolieri, R., Olesen, H.R., Ketzel, M., Berkowicz, R., Franke, J., Schatzmann, M., Schlunzen, K., Leitl, B., Britter, R., Borrego, C., Costa, A., Castelli, S., Reisin, T., Hellsten, A., Saloranta, J., Moussiopoulos, N., Barmpas, F., Brzozowski, K., Goricsan, I., Balczo, M., Bartzis, J., Efthimiou, G., Santiago, J., Martilli, A., Piringer, M., Baumann-Stanzer, K., Hirtl, M., Baklanov, A., Nuterman, R., Starchenko, A.: COST 732 in practice: the MUST model evaluation exercise. Int J Environ Pollut 44, 403-418 (2011)

48. Schatzmann, M., Britter, R.: Quality assurance and improvement of micro-scale meteorological models. Int J Environ Pollut 44, 139-146 (2011)

49. Schlegel, F., Stiller, J., Bienert, A., Maas, H.G., Queck, R., Bernhofer, C.: Large-eddy simulation of inhomogeneous canopy flows using high resolution terrestrial laser scanning data. J Fluid Mech 142, 223-243 (2012)

50. Schumann, U.: Subgrid scale model for finite difference simulations of turbulent flows in plane channels and annuli. J Comput Phys 18, 376-404 (1975)

51. Seifert, A., Heus, T., Pincus, R., Stevens, B.: Large-eddy simulation of the transient and near-equilibrium behavior of precipitating shallow convection. J Adv Model Earth Sy 7, 1918-1937 (2015)

52. Shaw, R.H., Schumann, U.: Large-eddy simulation of turbulent flow above and within a forest. BoundLay Meteorol 61(1), 47-64 (1992)

53. Shaw, R.H., Tavangar, J., Ward, D.P.: Structure of Reynolds stress in a canopy. J Clim Appl Meteorol 22, 1922-1931 (1983)

54. Smagorinsky, J.: General circulation experiments with the primitive equations: I. The basic experiment. Mon Weather Rev 91, 99-164 (1963)

55. Stull, R.B.: An Introduction to Boundary Layer Meteorology. Kluwer Academic Publishers, Dordrecht (1988)

56. Torrence, C., Compo, G.P.: A practical guide to wavelet analysis. B Am Meteorol Soc 79, 61-78 (1998) 
57. Townsend, A.A.: The Structure of Turbulent Shear Flow. Cambridge University Press, Cambridge (1956)

58. Trini Castelli, S., Baumann-Stanzer, K., Leitl, B., Milliez, C.M., Berbekar, E., Rakai, A., Fuka, V., Hellsten, A., Petrov, A., Efthimiou, G., Andronopoulos, S., Tinarelli, G., Tavares, R., Armand, P., Gariazzo, C., Jurcakova, K., Gašparac, G.: Evaluation of local-scale models for accidental releases in built environments: Results of the modelling exercises in cost action es1006. In: G.D. Steyn, N. Chaumerliac (eds.) Air Pollution Modeling and its Application XXIV, pp. 497-502. Springer International Publishing (2016)

59. Wallace, J.M.: Quadrant analysis in turbulence research: history and evolution. Annu Rev Fluid Mech 48, 131-158 (2016)

60. Wallace, J.M., Eckelmann, H., Brodkey, R.: The wall region in turbulent shear flow. J Fluid Mech 53, 39-48 (1972)

61. Willmarth, W.W., Lu, S.S.: Structure of the Reynolds stress near the wall. J Fluid Mech 55, 65-92 (1972)

62. Wood, C.R., Barlow, J.F., Belcher, S.E., Dobre, A., Arnold, S.J., Balogun, A.A., Lingard, J.J.N., Smalley, R.J., Tate, J.E., Tomlin, A.S., Britter, R.E., Cheng, H., Martin, D., Petersson, F.K., Shallcross, D.E., White, I.R., Neophytou, M.K., Robins, A.G.: Dispersion experiments in central London: The 2007 DAPPLE project. B Am Meteorol Soc 90, 955-969 (2009)

63. Wyngaard, J.C., Coté, O.R.: Cospectral similarity in the atmospheric surface layer. Q J Roy Meteorol Soc 98, 590-603 (1972)

64. Yaglom, A.M.: Correlation Theory of Stationary and Related Random Functions I: Basic Results. Springer (1987)

65. Yue, W., Parlange, M.B., Meneveau, C., Zhu, W., van Hout, R., Katz, J.: Large-eddy simulation of plant canopy flows using plant-scale representation. Bound-Lay Meteorol 124, 183-203 (2007) 